\title{
BVRI LIGHT CURVES FOR 29 TYPE Ia SUPERNOVAE
}

\section{MARIO HAMUY}

National Optical Astronomy Observatories, ${ }^{1}$ Cerro Tololo Inter-American Observatory, Casilla 603 , La Serena, Chile and University of Arizona, Steward Observatory, Tucson, Arizona 85721

Electronic mail: mhamuy@as.arizona.edu

M. M. Phillips, Nicholas B. SuntZeFF, ANd R. A. SCHOMmER

National Optical Astronomy Observatories, ${ }^{1}$ Cerro Tololo Inter-American Observatory, Casilla 603, La Serena, Chile Electronic mail: mphillips@noao.edu, nsuntzeff@noao.edu, rschommer@noao.edu

JOSÉ MAZA, ${ }^{2}$ ROBERTO ANTEZANA, AND MARINA WISCHNJEWSKY

Departamento de Astronomía, Universidad de Chile, Casilla 36-D, Santiago, Chile Electronic mail: jose@calan.das.uchile.cl

Geraldo Valladares, César Muena, L. E. González, R. Avilés, L. A. Wells, ${ }^{3}$ R. C. Smith, Mauricio Navarrete, Ricardo Covarrubias, G. M. Williger, ${ }^{4}$ Alistair R. Walker,

A. C. Layden, J. H. Elias, J. A. Baldwin, Manuel Hernández, Hernán TiRado, Patricio Ugarte, R. ELSTON, AND NELSON SAAVEDRA

National Optical Astronomy Observatories, ${ }^{1}$ Cerro Tololo Inter-American Observatory, Casilla 603, La Serena, Chile

Felipe Barrientos, Edgardo Costa, Paulina Lira, M. T. Ruiz, Claudio Angutta, Ximena Gómez, AND P. ORTIZ

Departamento de Astronomía, Universidad de Chile, Casilla 36-D, Santiago, Chile

M. Della Valle, J. Danziger, AND J. Storm

La Silla Observatory, European Southern Observatory, Casilla 19001, Santiago, Chile

Y-C. Kim, C. Bailyn, E. P. Rubenstein, D. TuCKer, ${ }^{5}$ S. Cersosimo, R. A. MÉndez, ${ }^{6}$ L. Siciliano, W. SHERRY, ${ }^{7}$ BRIAN CHABOYER, AND R. A. KOOPMANN

Yale University, Department of Astronomy, P.O. Box 208101, New Haven, Connecticut 06520-8101

D. GEISLER, A. SARAJEDINI, AND ARJUn DEY

National Optical Astronomy Observatories, ${ }^{1}$ Kitt Peak National Observatory, P.O. Box 26732, Tucson, Arizona 85726

\section{N. TYSON}

Department of Astrophysics Sciences, Princeton University, Peyton Hall, Princeton, New Jersey 08544

R. MICHAEL RICH AND R. GAL ${ }^{8}$

Department of Astronomy, Columbia University, Mail Code 5242, New York, New York 10027

\section{ROBERT LAMONTAGNE}

Département de Physique and Observatoire du mont Mégantic, Université de Montréal, C.P. 6128, Succ. Centre-Ville, Montréal, Canada

\section{N. CALDWELL}

Fred Lawrence Whipple Observatory, P.O. Box 97, 670 Mt. Hopkins Road, Amado, Arizona 85645

\section{PURAGRA GUHATHAKURTA}

UCO/Lick Observatory, University of California, Santa Cruz, California 95064

A. C. PHLLIPS ${ }^{9}$ AND P. SZKODY

Department of Astronomy, University of Washington, Seattle, Washington 98195

C. Prosser And Luis C. Ho

Harvard-Smithsonian Center for Astrophysics, 60 Garden Street, Cambridge, Massachusetts 02138 


\section{R. MCMAHAN}

Department of Physics and Astronomy, University of North Carolina, CB\# 3255 Phillips Hall, Chapel Hill, North Carolina 27599-3255

\section{G. BAGGLEY}

Department of Astrophysics, University of Oxford, Keble Road, Oxford, OX1 3RH, England, United Kingdom

\section{K.-P. CHENG}

California State University, Fullerton, Department of Physics, Fullerton, California 92634

\section{R. HAVLEN}

Astronomical Society of the Pacific, 390 Ashton Avenue, San Francisco, California 94112

\section{K. WAKAMATSU}

Physics Department, Gifu University, Gifu, 501-11, Japan

\section{K. JANES}

Department of Astronomy, Boston University, 725 Commonwealth Avenue, Boston, Massachusetts 02215

\section{MALKAN AND F. BAGANOFF}

Division of Astronomy, University of California Los Angeles, California 90095-1562

\section{P. SEITZER AND M. SHARA}

Space Telescope Science Institute, 3700 San Martin Drive, Baltimore, Maryland 21218

C. STURCH

Computer Sciences Corporation, Space Telescope Science Institute, Homewood Campus, Baltimore, Maryland 21218

\section{J. HESSER}

Dominion Astrophysical Observatory, 5071 West Saanich Road, R.R. 5, Victoria, BC V8X 4M6, Canada

\section{P. HARTIGAN ${ }^{10}$ AND J. HUGHES}

Five College Astronomy Department, University of Massachusetts, Amherst, Massachusetts 01003-4525

$$
\text { D. WELCH }
$$

Department of Physics and Astronomy, McMaster University, Hamilton, Ontario L8S 4M1, Canada

\section{T. B. WILLIAMS}

Department of Physics and Astronomy, Rutgers University, P.O. Box 849, Piscataway, New Jersey 08855-0849

\section{H. FERGUSON ${ }^{11}$}

Institute of Astronomy, University of Cambridge, Madingley Road, Cambridge, CB3 OHA, England, United Kingdom

\section{P. J. FRANCIS}

School of Physics, University of Melbourne, Parkville, Victoria 3052, Australia

\section{FRENCH}

Wheelock College, 200 The Riverway, Boston, Massachusetts 02215

\section{BOLTE}

Lick Observatory, University of California, Santa Cruz, California 95064

\section{J. ROTH}

Sky Publishing Corporation, 49 Bay State Road, Cambridge, Massachusetts 02138

\section{S. ODEWAHN}

School of Physics and Astronomy, University of Minnesota 116 Church Street, SE, Minneapolis, Minnesota 55455

\section{(C) American Astronomical Society - Provided by the NASA Astrophysics Data System}




\author{
S. HOWELL \\ Department of Physics and Astronomy, University of Wyoming, Laramie, Wyoming 82071 \\ W. KRZEMINSKI \\ Carnegie Institution of Washington, Las Campanas Observatory, Casilla 601, La Serena, Chile \\ Received 1996 June 26; revised 1996 September 5
}

\begin{abstract}
$B V(R I)_{K C}$ light curves are presented for 27 type Ia supernovae discovered during the course of the Calán/Tololo Survey and for two other SNe Ia observed during the same period. Estimates of the maximum light magnitudes in the $B, V$, and $I$ bands and the initial decline rate parameter $\Delta m_{15}(B)$ are also given. (C) 1996 American Astronomical Society.
\end{abstract}

\section{INTRODUCTION}

The Calán/Tololo Supernova Survey was begun in 1990 as a collaboration between astronomers at Cerro Tololo Inter-American Observatory (CTIO) and the Cerro Calán Observatory of the University of Chile with the principal goal of examining the Hubble diagram for type Ia supernovae (SNe Ia) out to redshifts of $\sim 0.1$. During the course of the survey, which we completed in 1993 November, a total of $32 \mathrm{SNe}$ Ia were discovered and spectroscopically confirmed. Of these, useful follow-up CCD photometry was obtained for 27 events. In addition, as part of the same program, light curves were obtained of $2 \mathrm{SNe}$ Ia discovered at other observatories.

In this paper, we present the final reduced $B V R I$ light curves for these $29 \mathrm{SNe}$ Ia, along with estimates of the maximum-light magnitudes in $B V I$ and the initial decline rate parameter $\Delta m_{15}(B)$ (Phillips 1993). Note that preliminary light curves for a few events have appeared in previous publications (Hamuy et al. 1993a, hereafter referred to as Paper I; Maza et al. 1994, hereafter referred to as Paper II; Hamuy

${ }^{1}$ Cerro Tololo Inter-American Observatory, Kitt Peak National Observatory, National Optical Astronomy Observatories, operated by the Association of Universities for Research in Astronomy, Inc., (AURA), under cooperative agreement with the National Science Foundation.

${ }^{2}$ Cátedra Presidencial de Ciencias (Chile), 1996-1997.

${ }^{3}$ Current address: University of Arizona, Steward Observatory, Tucson, Arizona 85721.

${ }^{4}$ Current address: Max-Planck-Institut für Astronomie, Königstuhl 17, D-69117 Heidelberg, Germany.

${ }^{5}$ Current address: Astrophysikalisches Institut Potsdam, An der Sternwarte 16, D-14482 Potsdam, Germany.

${ }^{6}$ Current address: European Southern Observatory, Karl-SchwarzschildStraße 2, D-85748, Garching b. München, Germany.

${ }^{7}$ Current address: Astronomy Program, S.U.N.Y. at Stony Brook, Stony Brook, New York.

${ }^{8}$ Current address: Caltech, Mail Code 105-24, Pasadena, CA 91125.

${ }^{9}$ Current address: Lick Observatory, University of California at Santa Cruz, Santa Cruz, CA 95064.

${ }^{10}$ Current address: Department of Space Physics and Astronomy Rice University, Houston TX 77251-1892.

${ }^{11}$ Current address: Space Telescope Science Institute, 3700 San Martin Drive, Baltimore, MD 21218. et al. 1994, hereafter referred to as Paper III). In two accompanying papers, we use these data to examine (1) the absolute luminosities of the sample (Hamuy et al. 1996a, hereafter referred to as Paper V) and (2) the Hubble diagrams in $B V I$ and the value of the Hubble constant (Hamuy et al. 1996 b hereafter referred to as Paper VI).

\section{OBSERVATIONS}

The search phase of the Calán/Tololo Supernova Survey consisted of photographic observations of 45 fields taken with the Curtis Schmidt Camera, with observations carried out approximately twice a month over the 1990-93 period. The details of these observations were described in considerable detail in Paper I, and therefore will not be repeated here. The follow-up phase consisted of two parts: (1) classification via optical spectroscopy, and (2) photometric monitoring via direct CCD imaging in the $B V(R I)_{K C}$ system. Of the $50 \mathrm{SNe}$ discovered in the course of the Survey for which classification spectra were obtained, $32(64 \%)$ were found to be type Ia events. A complete listing of these SNe Ia is found in Table 1 which gives: the SN and host galaxy names, the morphology and heliocentric redshift of the host galaxy; the line-of-sight extinction due to our own Galaxy (Burstein \& Heiles 1982); the SN equatorial coordinates derived from an early-epoch CCD image using reference stars measured from the digitized sky survey plates available from the Space Telescope Science Institute; the offset of the SN from the host galaxy nucleus; the estimated photographic magnitude of the SN on the discovery plate; the name of the discoverer; and the UT discovery date. A $V$ band CCD image of each SN is reproduced in Fig. 1.

Follow-up photometry was obtained for as many of these events as proved practical. Spectra of three of the SNe (1992O, 1992ai, and 1993af) showed that these had been caught several weeks or months past maximum light; hence, 
TABLE 1. Type Ia supernovae.

\begin{tabular}{|c|c|c|c|c|c|c|c|c|c|c|c|}
\hline $\mathrm{SN}$ & $\begin{array}{c}\text { Host } \\
\text { Galaxy }\end{array}$ & $\begin{array}{l}\text { Morph. } \\
\text { Type }\end{array}$ & $z_{\text {helio }}$ & $\begin{array}{c}\mathrm{E}(B-V) \\
\pm 0.015\end{array}$ & $\begin{array}{c}\mathrm{SN} \\
\alpha(2000)\end{array}$ & $\begin{array}{c}\text { SN } \\
\delta(2000)\end{array}$ & \multicolumn{2}{|c|}{$\begin{array}{l}\text { Offset } \\
( \pm 0.1)\end{array}$} & $\begin{array}{c}m_{p g} \\
( \pm 0.5)\end{array}$ & Discoverer & $\begin{array}{l}\text { Date } \\
\text { (UT) }\end{array}$ \\
\hline \multicolumn{12}{|c|}{ Calán/Tololo } \\
\hline $1990 \mathrm{~T}$ & PGC 63925 & $\mathrm{Sa}$ & 0.0404 & 0.030 & $19: 59: 02.28$ & $-56: 15: 30.0$ & $24.8 \mathrm{E}$ & $1.9 \mathrm{~S}$ & 16.5 & Antezana & Jul 21.11 \\
\hline 1990Y & anonymous & $\mathrm{E}$ ? & 0.0391 & 0.000 & $03: 37: 22.64$ & $-33: 02: 40.1$ & $1.0 \mathrm{E}$ & $5.0 \mathrm{~S}$ & 17.8 & Wischnjewsky & Aug 22.36 \\
\hline 1990af & anonymous & SB0 & 0.0506 & 0.015 & $21: 34: 58.12$ & $-62: 44: 07.4$ & $8.0 \mathrm{~W}$ & $7.4 \mathrm{~N}$ & 16.8 & Antezana & Oct 24.07 \\
\hline $1991 \mathrm{~S}$ & UGC 5691 & $\mathrm{Sb}$ & 0.0546 & 0.000 & $10: 29: 27.79$ & $+22: 00: 46.4$ & $4.4 \mathrm{E}$ & $17.3 \mathrm{~N}$ & 17.5 & Antezana & Apr 10.09 \\
\hline $1991 \mathrm{U}$ & IC 4232 & $\mathrm{Sbc}$ & 0.0317 & 0.070 & $13: 23: 22.20$ & $-26: 06: 28.7$ & $2.2 \mathrm{~W}$ & $5.8 \mathrm{~N}$ & 16.0 & Antezana & Apr 18.20 \\
\hline 1991ag & IC 4919 & $\mathrm{SBb}$ & 0.0141 & 0.015 & $20: 00: 08.65$ & $-55: 22: 03.4$ & $4.4 W$ & $22.1 \mathrm{~N}$ & 15.0 & Antezana & Jun 9.19 \\
\hline $1992 \mathrm{~J}$ & anonymous & $\mathrm{E} / \mathrm{S} 0$ & 0.0446 & 0.050 & 10:09:00.30 & $-26: 38: 24.4$ & $11.9 \mathrm{~W}$ & $12.0 \mathrm{~N}$ & 18.5 & Wischnjewsky & Mar 3.15 \\
\hline $1992 \mathrm{~K}$ & ESO 269-57 & $\mathrm{SBb}$ & 0.0103 & 0.120 & $13: 10: 04.20$ & $-46: 26: 30.3$ & $1.9 \mathrm{~W}$ & $15.4 \mathrm{~S}$ & 17.5 & Antezana & Mar 3.32 \\
\hline 19920 & anonymous & $?$ & 0.037 & 0.045 & $19: 23: 42.29$ & $-62: 49: 30.1$ & $7.0 \mathrm{~W}$ & $1.0 \mathrm{~N}$ & 18.5 & Antezana & Mar 10.37 \\
\hline $1992 \mathrm{P}$ & IC 3690 & $\mathrm{SBa}$ & 0.0252 & 0.018 & $12: 42: 48.95$ & $+10: 21: 37.5$ & $4.3 \mathrm{~W}$ & $9.8 \mathrm{~N}$ & 16.8 & Antezana & Apr 3.20 \\
\hline $1992 \mathrm{ae}$ & anonymous & E1? & 0.0752 & 0.015 & $21: 28: 17.66$ & $-61: 33: 00.0$ & $2.1 \mathrm{E}$ & $4.0 \mathrm{~N}$ & 18.0 & Antezana & Jun 27.30 \\
\hline $1992 \mathrm{ag}$ & ESO 508-67 & $S$ & 0.0249 & 0.060 & $13: 24: 10.12$ & $-23: 52: 39.3$ & $\cdots$ & $\cdots$ & 16.5 & Wischnjewsky & Jun 29.13 \\
\hline 1992ai & anonymous & $?$ & $\cdots$ & 0.000 & 01:29:08.04 & $-32: 16: 30.0$ & $2.5 \mathrm{~W}$ & $7.0 \mathrm{~S}$ & 17.5 & Antezana & Jul 5.42 \\
\hline $1992 \mathrm{aq}$ & anonymous & $\mathrm{Sa} ?$ & 0.1018 & 0.000 & $23: 04: 34.76$ & $-37: 20: 42.1$ & $2.4 \mathrm{E}$ & $7.1 \mathrm{~S}$ & 19.0 & Antezana & Jul 26.22 \\
\hline $1992 \mathrm{au}$ & anonymous & $\mathrm{E} 1$ & 0.0614 & 0.000 & $00: 10: 40.27$ & $-49: 56: 43.3$ & $21.2 \mathrm{E}$ & $8.9 \mathrm{~N}$ & 17.0 & Antezana & Jul 29.25 \\
\hline $1992 b c$ & ESO $300-9$ & Sab & 0.0202 & 0.000 & $03: 05: 17.28$ & $-39: 33: 39.7$ & $16.1 \mathrm{E}$ & $2.0 \mathrm{~S}$ & 15.0 & Antezana & Oct 4.36 \\
\hline $1992 \mathrm{bg}$ & anonymous & $\mathrm{Sa}$ & 0.0352 & 0.170 & $07: 41: 56.53$ & $-62: 31: 08.8$ & $3.4 \mathrm{~W}$ & $5.8 \mathrm{~N}$ & 17.8 & Antezana & Oct 21.34 \\
\hline $1992 \mathrm{bh}$ & anonymous & $\mathrm{Sbc}$ & 0.0450 & 0.000 & $04: 59: 27.55$ & $-58: 49: 44.2$ & $1.9 \mathrm{E}$ & $3.6 \mathrm{~S}$ & 17.3 & Antezana & Oct 21.32 \\
\hline $1992 \mathrm{bk}$ & ESO $156-8$ & $\mathrm{E} 1$ & 0.0581 & 0.000 & 03:43:01.90 & $-53: 37: 56.8$ & $11.9 \mathrm{E}$ & $21.1 \mathrm{~N}$ & 18.0 & Wischnjewsky & Nov 19.23 \\
\hline $1992 \mathrm{bl}$ & ESO 291-11 & $\mathrm{SB} 0 / \mathrm{SBa}$ & 0.0437 & 0.000 & $23: 15: 13.25$ & $-44: 44: 34.5$ & $15.3 \mathrm{E}$ & $21.8 \mathrm{~S}$ & 16.8 & Antezana & Nov 20.07 \\
\hline 1992bo & ESO $352-57$ & $\mathrm{E} 5 / \mathrm{S} 0$ & 0.0189 & 0.000 & $01: 21: 58.44$ & $-34: 12: 43.5$ & $47.3 \mathrm{~W}$ & 54.75 & 17.0 & Antezana & Dec 20.06 \\
\hline $1992 b p$ & anonymous & $\mathrm{E} 2 / \mathrm{S} 0$ & 0.0793 & 0.040 & $03: 36: 37.95$ & $-18: 21: 13.7$ & $5.4 \mathrm{~W}$ & $1.4 \mathrm{~S}$ & 18.0 & Wischnjewsky & Dec 20.16 \\
\hline $1992 \mathrm{br}$ & anonymous & $\mathrm{E} 0$ & 0.0882 & 0.000 & $01: 45: 44.83$ & $-56: 05: 57.9$ & $3.6 \mathrm{E}$ & $6.3 \mathrm{~S}$ & 18.5 & Wischnjewsky & Dec 27.09 \\
\hline $1992 \mathrm{bs}$ & anonymous & $\mathrm{SBb}$ & 0.0637 & 0.000 & $03: 29: 27.20$ & $-37: 16: 18.9$ & $9.0 \mathrm{~W}$ & $3.6 \mathrm{~N}$ & 18.0 & González & Dec 28.17 \\
\hline 1993B & anonymous & $\mathrm{SBb}$ & 0.0696 & 0.080 & $10: 34: 51.38$ & $-34: 26: 30.0$ & $0.9 \mathrm{E}$ & $5.4 \mathrm{~N}$ & 18.0 & Antezana & $\operatorname{Jan} 17.22$ \\
\hline $1993 \mathrm{H}$ & ESO 445-66 & $\mathrm{SBb}(\mathrm{rs})$ & 0.0239 & 0.040 & $13: 52: 50.34$ & $-30: 42: 23.3$ & $1.0 \mathrm{E}$ & $12.3 \mathrm{~N}$ & 16.5 & Wischnjewsky & Mar 18.20 \\
\hline $1993 \mathrm{M}$ & anonymous & $?$ & 0.090 & 0.045 & $19: 13: 01.53$ & $-64: 17: 28.3$ & $1.0 \mathrm{~W}$ & $3.0 \mathrm{~S}$ & 18.5 & Antezana & Apr 28.33 \\
\hline 19930 & anonymous & E5/S01 & 0.0510 & 0.035 & $13: 31: 07.87$ & $-33: 12: 50.5$ & $14.1 \mathrm{~W}$ & $8.4 \mathrm{~N}$ & 17.5 & Antezana & May 18.16 \\
\hline $1993 \mathrm{~T}$ & anonymous & $?$ & 0.088 & 0.000 & $23: 10: 54.09$ & $-44: 58: 48.6$ & $18.5 \mathrm{~W}$ & $13.5 \mathrm{~N}$ & 18.0 & Antezana & May 26.38 \\
\hline 1993af & NGC 1808 & $\mathrm{Sbc}$ & 0.0034 & 0.015 & 05:08:00.71 & $-37: 29: 18.0$ & $220.0 \mathrm{E}$ & $94.0 \mathrm{~N}$ & 17.0 & Wischnjewsky & Nov 15.30 \\
\hline 1993ag & anonymous & E3/S01 & 0.0490 & 0.150 & $10: 03: 35.00$ & $-35: 27: 47.6$ & $5.0 \mathrm{~W}$ & $6.1 \mathrm{~S}$ & 18.5 & Antezana & Nov 15.33 \\
\hline 1993ah & ESO 471-27 & $\mathrm{S} 02$ & 0.0297 & 0.000 & $23: 51: 50.27$ & $-27: 57: 47.0$ & $0.9 \mathrm{~W}$ & $8.1 \mathrm{~N}$ & 17.0 & Wischnjewsky & Nov 16.13 \\
\hline \multicolumn{12}{|c|}{ Other } \\
\hline 19900 & PGC 59955 & $\mathrm{SBa}$ & 0.0303 & 0.070 & $17: 15: 35.92$ & $+16: 19: 25.8$ & $21.8 \mathrm{E}$ & $3.9 \mathrm{~S}$ & 17.0 & Mueller & Jun 22 \\
\hline 1992al & ESO 234-69 & $\mathrm{Sb}$ & 0.0146 & 0.000 & $20: 45: 56.45$ & $-51: 23: 40.0$ & $19.0 \mathrm{E}$ & $12.0 \mathrm{~S}$ & 16.0 & McNaught & Jul 27.6 \\
\hline
\end{tabular}

the decision was made not to concentrate on obtaining follow-up photometry of these events. For two of the more distant SNe, 1993M and 1993T, an insufficient number of observations were secured to provide adequate coverage of the light curves. Hence, the final number of Calán/Tololo SNe Ia for which light curves were ultimately obtained was 27.

In addition to these $27 \mathrm{SNe}$, we obtained $B V R I$ photometry of two other SNe Ia, 19900 and 1992al, which were discovered at other observatories during the course of the survey. Information for these two events is included at the end of Table 1, and $V$ band CCD images are shown in Fig. 2. Thus, in this paper we present light curves for a total of 29 SNe Ia.

The follow-up photometry for all 29 SNe Ia was obtained with CCD detectors on a total of 302 nights between 1990 July 4 and 1995 February 11 thanks to the extensive collaboration of many visiting astronomers and CTIO staff members. The vast majority (94\%) of these nights were at CTIO, with the remaining observations being carried out with the Las Campanas Observatory (LCO) 1.0-m telescope and four different telescopes at the European Southern Observatory (ESO). At CTIO, fully $90 \%$ of the data were taken with the 0.9-m telescope, with the remainder coming from the $1.5-\mathrm{m}$ and 4.0-m telescopes. ${ }^{12}$ A complete journal of the observations is given in Table 2, which contains the following information: the UT date, the telescope employed, the observatory, the identity of the CCD detector, and the observer(s).

\section{PHOTOMETRIC REDUCTIONS}

A detailed description of the procedures we followed to produce $B V R I$ magnitudes from the individual CCD images of each SN has been given in Paper III. The various steps are summarized as follows:

(1) Several deep CCD images (in each color) were obtained of the SN field after the SN had faded from detection. These images were transformed geometrically to the same scale, and then coadded to produce a deep master image of the host galaxy.

(2) The master galaxy image was transformed and scaled to the flux scale of each individual SN image, and then sub-

\footnotetext{
${ }^{12}$ This project serves as an eloquent illustration of the capabilities of "small" telescopes equipped with state-of-the-art CCD detectors.
} 

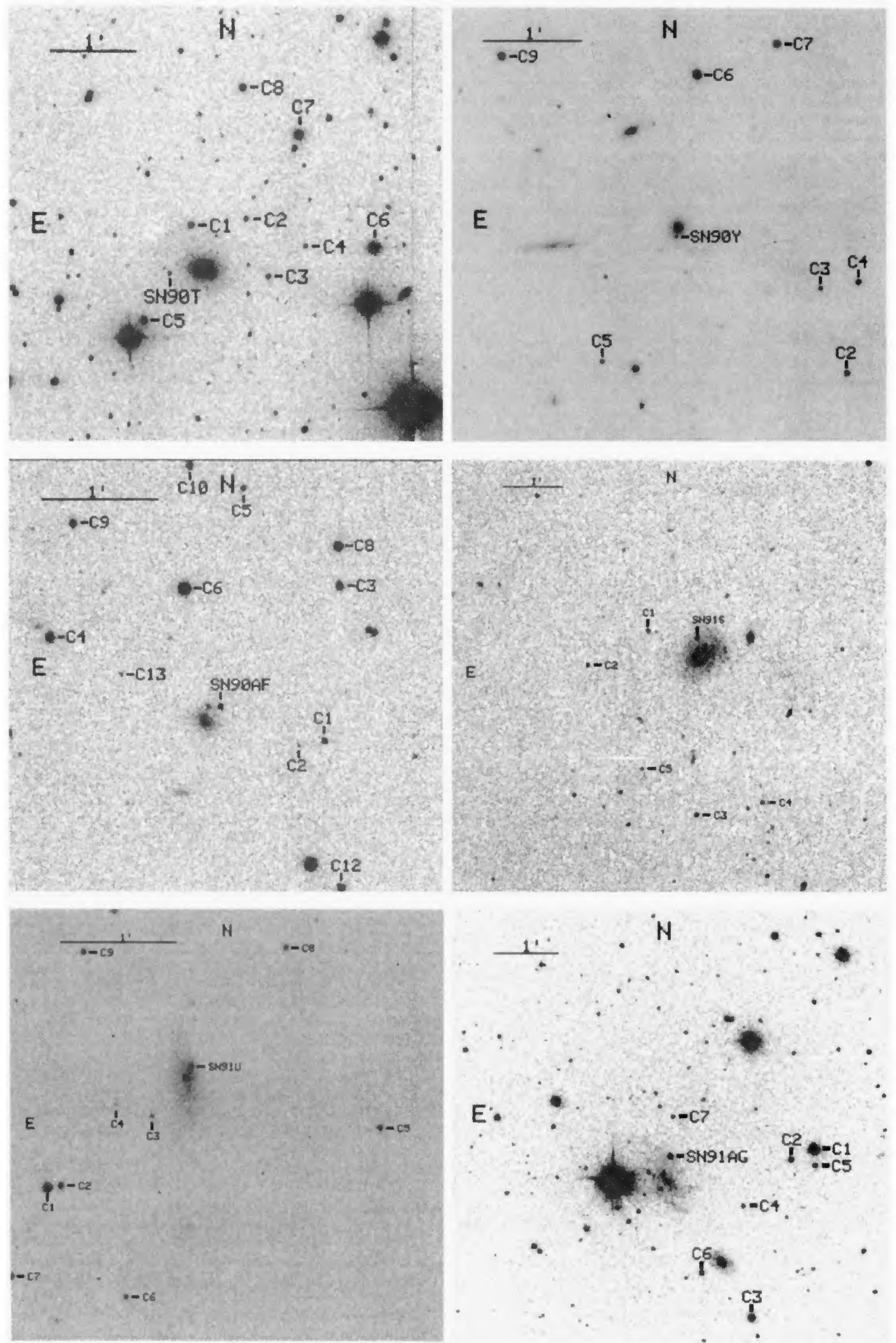

FIG. 1. $V$ band CCD images of the $32 \mathrm{SNe}$ Ia discovered in the course of the Calán/Tololo survey. The photometric sequence stars are labeled along with the SNe. 


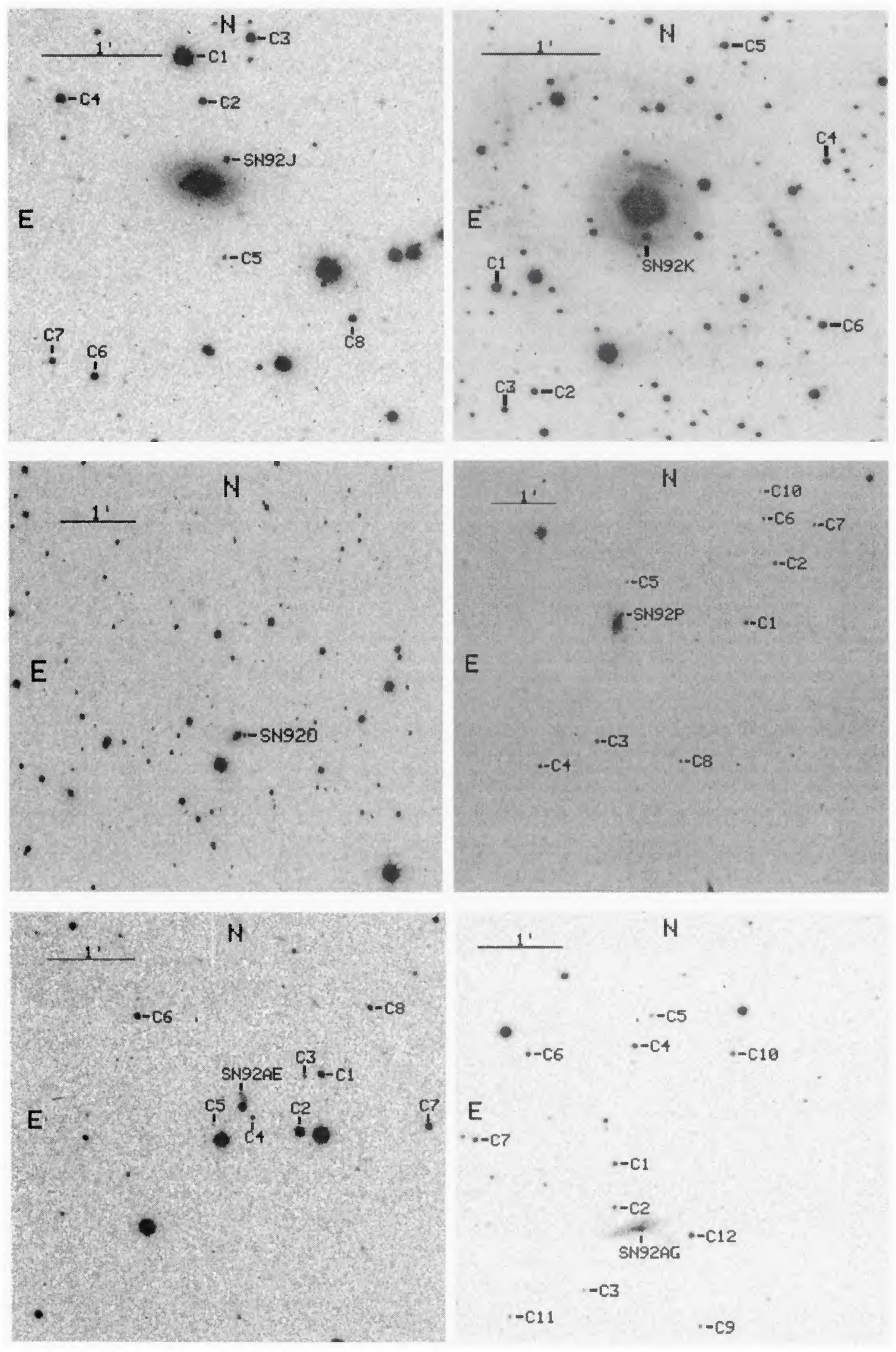

FIG. 1. (continued) 

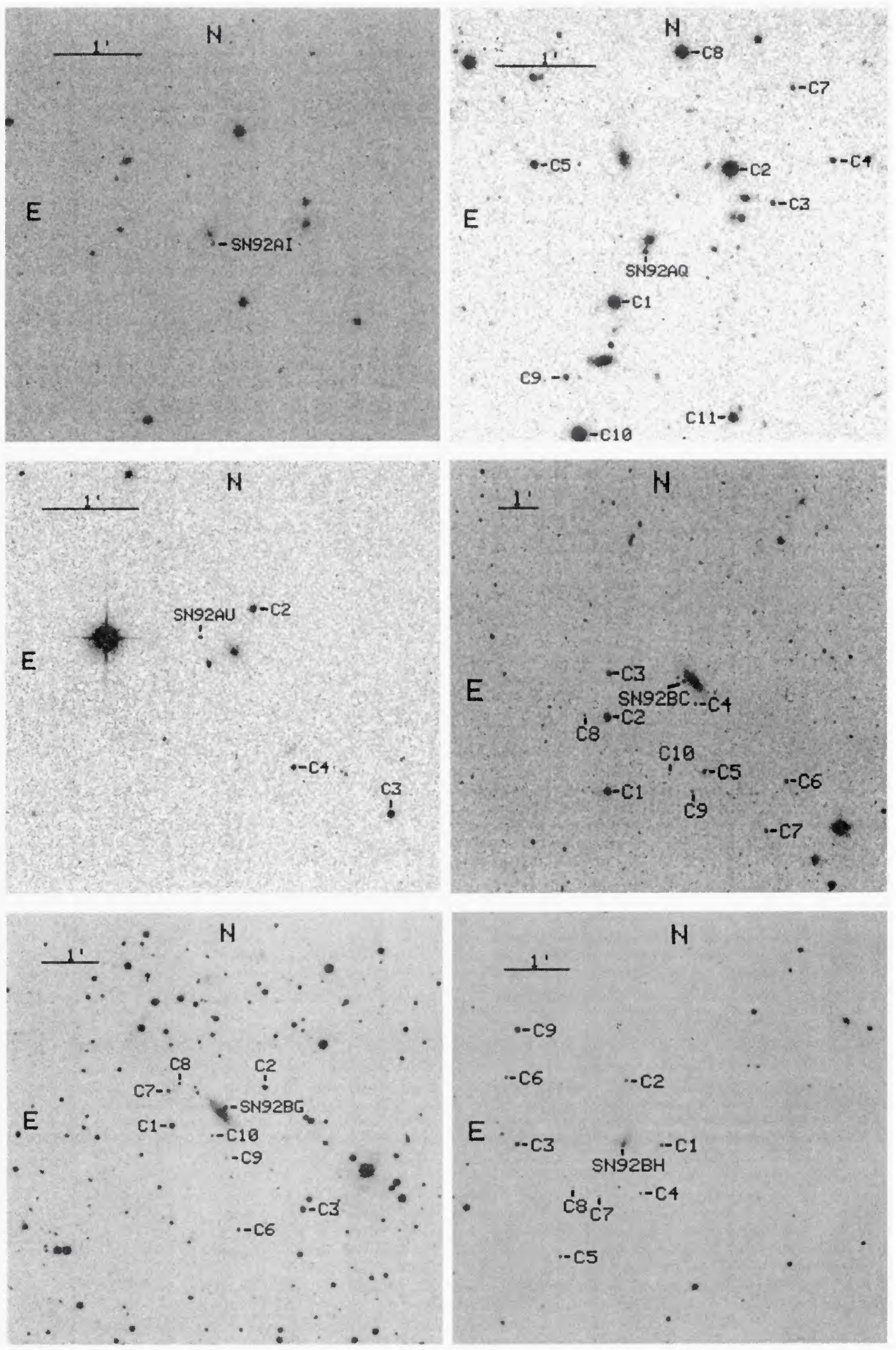

FiG. 1. (continued) 

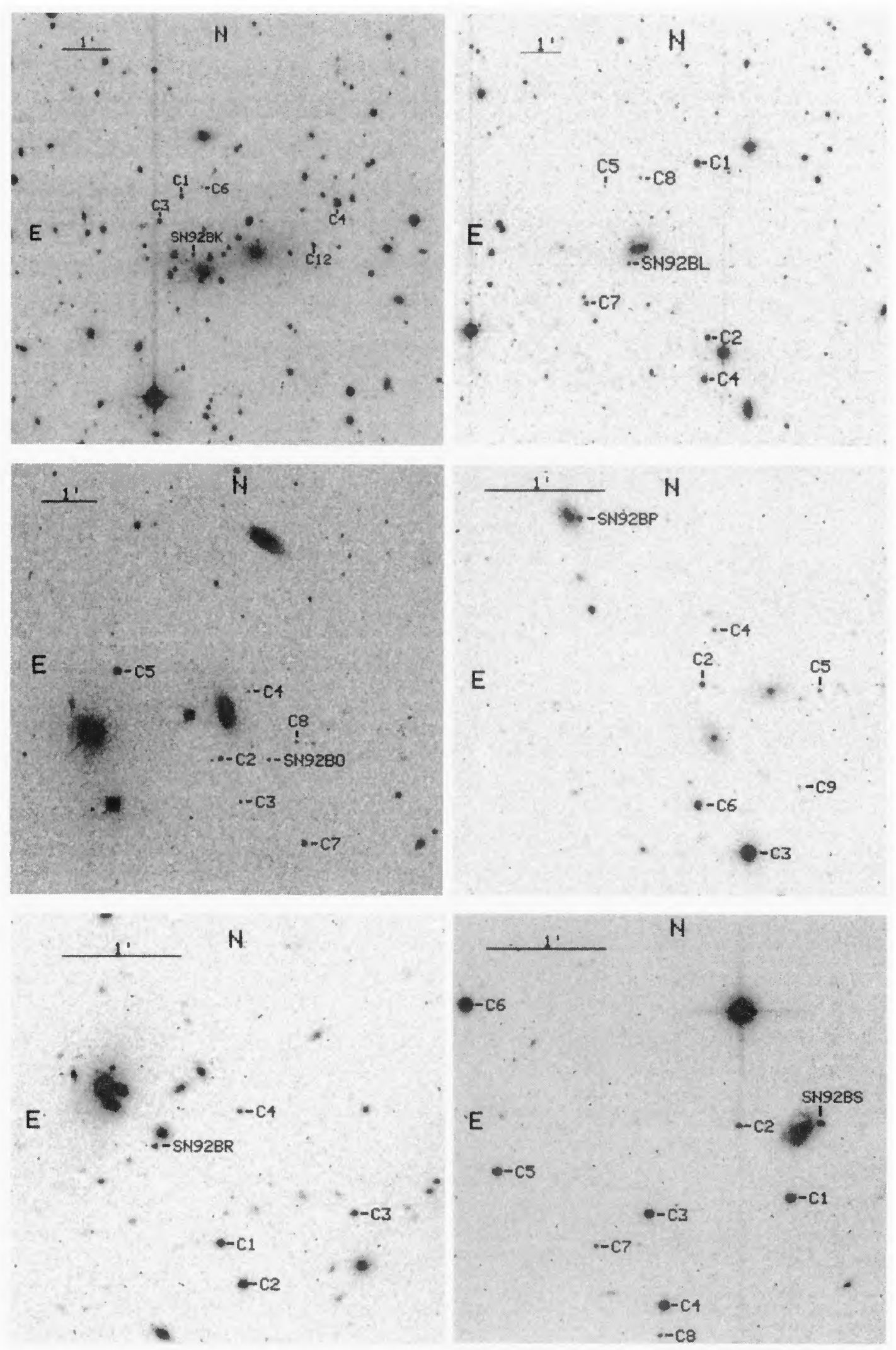

FIG. 1. (continued) 


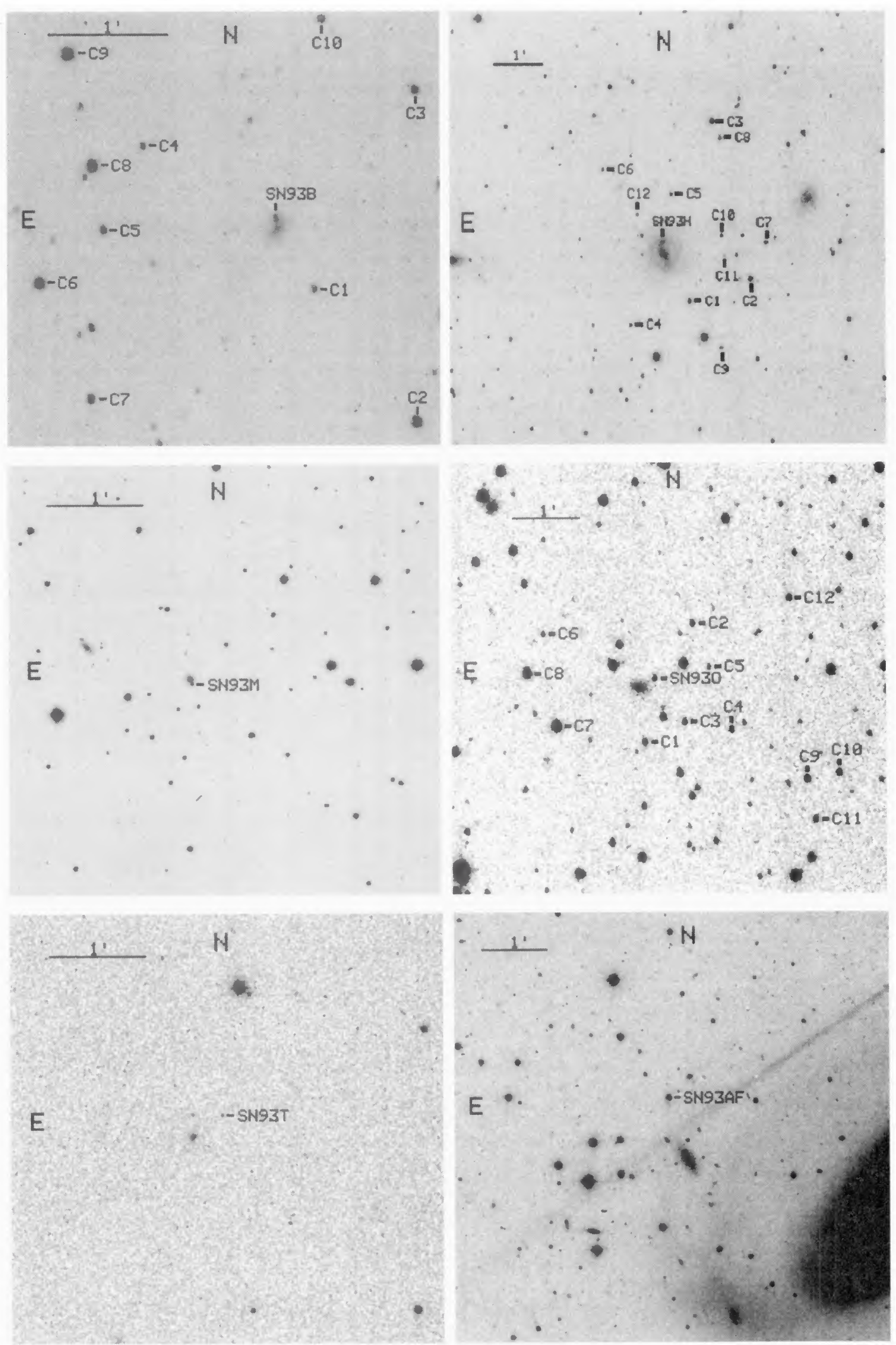

FIG. 1. (continued) 


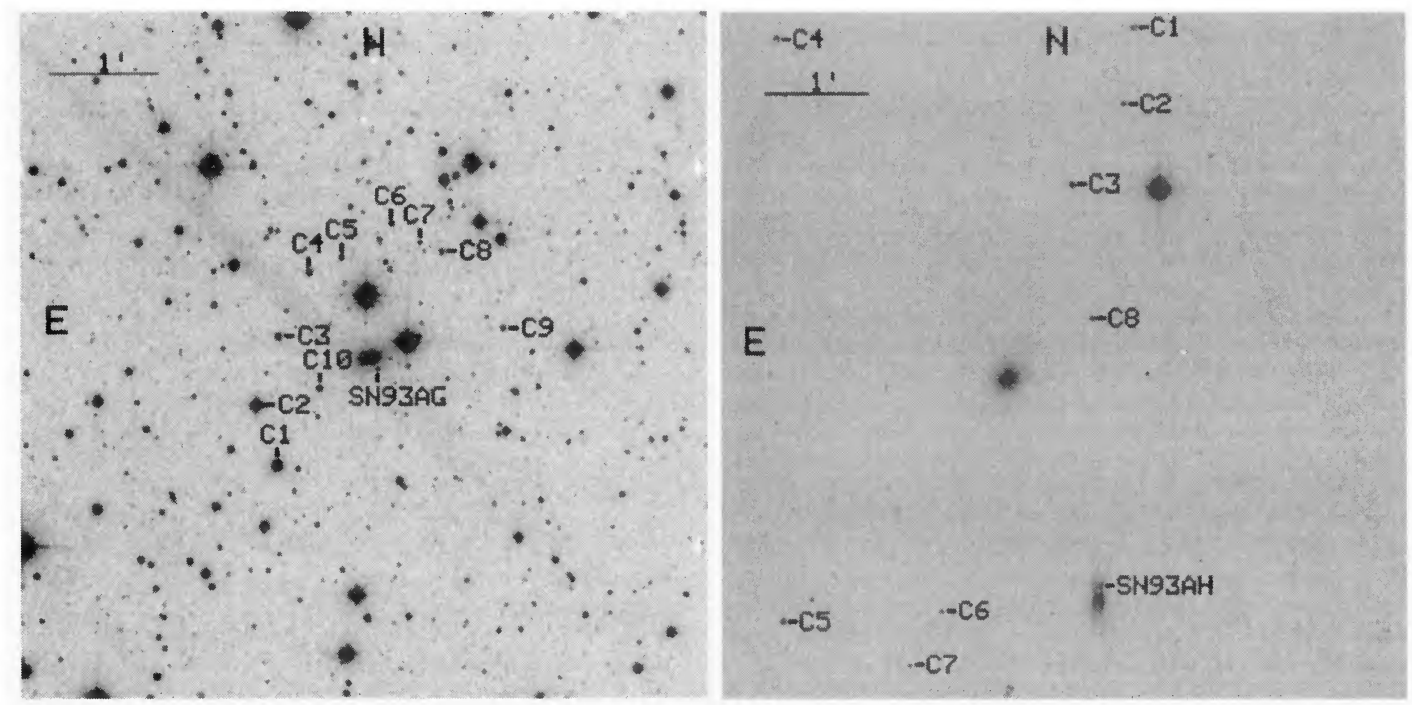

FIG. 1. (continued)

tracted. In order to save computing time, this galaxy subtraction was carried out over only a subset of the image centered on the SN (see Fig. 2 of Paper III).

(3) Instrumental magnitudes of the $\mathrm{SN}$ and several field local standard stars were then measured from the galaxysubtracted images via point spread function fitting.

(4) Finally, the instrumental magnitudes were transformed to the standard $B V(R I)_{K C}$ system through the use of a photometric sequence set up in the same field surrounding the SN. (See Paper I for further details of the exact photometric transformations employed.) The photometric sequences for all $29 \mathrm{SNe}$ are identified in the finder charts in Figs. 1 and 2. Only three stars lie outside the observed fields and could not be identified in these charts, namely: $c 8$ in the field of $\mathrm{SN}$ $1990 \mathrm{Y}$ which is located about 130 arcsec west from star $c 6$; $c 10$ in the field of SN 1990 Y which is located about 160 arcsec west and 35 arcsec south from star $c 6$; and $c 14$ in the field of SN 1990af which is located about 10 arcsec south and 40 arcsec east from star $c 12$. The magnitudes for the photometric sequences are listed in Table 3. In every case, these sequences were derived from observations made on several (typically 4-6) photometric nights. The uncertainties quoted correspond to the standard error of the mean.

Table 4 (this table can be found in the AAS CD-ROM Series, Vol. 7, 1996) lists the final reduced photometry for each SN. Please note that these magnitudes supersede all previously published values (Papers I, II, and III) for the same SNe. The uncertainties quoted for each magnitude correspond to the sum in quadrature of the errors due to photon Poisson statistics and an assumed additional error of $0.03^{m}$ in each individual observation. The latter uncertainty was included in order to account for errors involved in the transformation from our instrumental system to the standard sys-

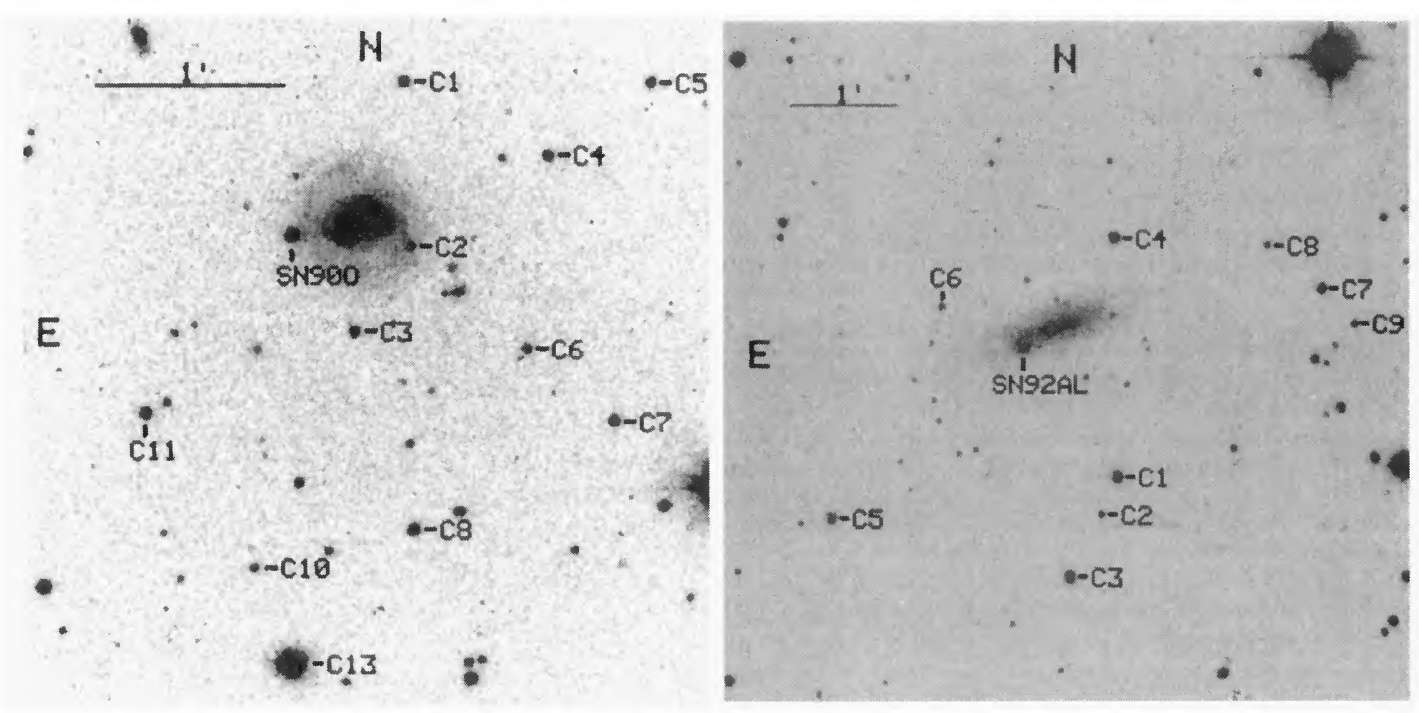

FIG. 2. $V$ band CCD images of the two SNe Ia, 19900 and 1992al, discovered at other observatories and included in the Calán/Tololo follow-up photometric program. The photometric sequence stars are labeled along with the SNe. 
TABLE 2. Journal of the observations.

\begin{tabular}{|c|c|c|c|c|}
\hline Date (UT) & Telescope & Observatory & $\mathrm{CCD}$ & Observer(s) \\
\hline $1990 \mathrm{Jul} 04$ & $0.91-\mathrm{m}$ & CTIO & TEK IV & N. Suntzeff \\
\hline 1990 Jul 12 & $0.91-\mathrm{m}$ & CTIO & TEK IV & $\cdots$ \\
\hline 1990 Jul 23 & $0.91-\mathrm{m}$ & CTIO & $\mathrm{TI} 2$ & M. Rich/N. Tyson \\
\hline 1990 Jul 26 & $0.91-\mathrm{m}$ & CTIO & TEK IV & T. Williams \\
\hline 1990 Jul 27 & $0.91-\mathrm{m}$ & CTIO & TI3 & S. Odewahn \\
\hline 1990 Jul 31 & $0.91-\mathrm{m}$ & CTIO & $\mathrm{TI3}$ & R. Guhathakurta \\
\hline 1990 Aug 01 & $0.91-\mathrm{m}$ & CTIO & TI3 & N. Suntzeff \\
\hline 1990 Aug 12 & $0.91-\mathrm{m}$ & CTIO & $\mathrm{TI} 3$ & L. Wells \\
\hline 1990 Aug 21 & $0.91-\mathrm{m}$ & CTIO & $\mathrm{TI} 3$ & J. Elias \\
\hline 1990 Aug 22 & $0.91-\mathrm{m}$ & CTIO & $\mathrm{TI} 3$ & J. Elias \\
\hline 1990 Aug 24 & $0.91-\mathrm{m}$ & CTIO & $\mathrm{TI} 3$ & M. Navarrete \\
\hline 1990 Aug 29 & $0.91-\mathrm{m}$ & CTIO & $\mathrm{TI3}$ & S. Howell/P. Szkody \\
\hline 1990 Aug 30 & $0.91-\mathrm{m}$ & CTIO & TI3 & C. Sturch \\
\hline 1990 Sep 01 & $0.91-\mathrm{m}$ & CTIO & $\mathrm{TI} 3$ & C. Sturch \\
\hline 1990 Sep 08 & $0.91-\mathrm{m}$ & CTIO & TI1 & H. Tirado \\
\hline 1990 Sep 10 & $1.0-\mathrm{m}$ & $\mathrm{LCO}$ & TI1 & J. Maza/F. Barrientos \\
\hline 1990 Sep 11 & $1.0-\mathrm{m}$ & $\mathrm{LCO}$ & TI1 & J. Maza/F. Barrientos \\
\hline 1990 Sep 13 & $1.0-\mathrm{m}$ & LCO & TI1 & J. Maza/F. Barrientos \\
\hline 1990 Sep 14 & $1.0-\mathrm{m}$ & LCO & TI1 & J. Maza/F. Barrientos \\
\hline 1990 Sep 15 & $0.91-\mathrm{m}$ & CTIO & $\mathrm{T} 12$ & M. Hernández \\
\hline 1990 Sep 20 & $0.91-\mathrm{m}$ & CTIO & $\mathrm{T} 12$ & J. Roth \\
\hline 1990 Sep 29 & $0.91-\mathrm{m}$ & CTIO & TI3 & $\cdots$ \\
\hline 1990 Oct 10 & $0.91-\mathrm{m}$ & CTIO & $\mathrm{TI} 2$ & N. Suntzeff \\
\hline 1990 Oct 14 & $4.0-\mathrm{m}$ & CTIO & TEK IV & M. Navarrete \\
\hline 1990 Oct 20 & $0.91-\mathrm{m}$ & CTIO & TI3 & L. González \\
\hline 1990 Oct 24 & $0.91-\mathrm{m}$ & CTIO & $\mathrm{TI} 3$ & L. González \\
\hline 1990 Oct 28 & $0.91-\mathrm{m}$ & CTIO & $\mathrm{TI3}$ & R. Lamontagne \\
\hline 1990 Oct 29 & $0.91-\mathrm{m}$ & CTIO & TI3 & R. Lamontagne \\
\hline 1990 Oct 30 & $0.91-\mathrm{m}$ & CTIO & $\mathrm{TI3}$ & R. Lamontagne \\
\hline 1990 Oct 31 & $0.91-\mathrm{m}$ & CTIO & $\mathrm{TI} 3$ & R. Lamontagne \\
\hline 1990 Nov 01 & $0.91-\mathrm{m}$ & CTIO & TEK IV & R. Schommer \\
\hline 1990 Nov 02 & 0.91-m & CTIO & $\mathrm{TI} 2$ & F. Baganoff \\
\hline 1990 Nov 04 & $0.91-\mathrm{m}$ & CTIO & $\mathrm{TI} 2$ & F. Bagnoff \\
\hline 1990 Nov 06 & $0.91-\mathrm{m}$ & CTIO & TEK IV & A. Phillips \\
\hline 1990 Nov 08 & $0.91-\mathrm{m}$ & CTIO & TEK IV & A. Phillips \\
\hline 1990 Nov 11 & $1.5-\mathrm{m}$ & CTIO & TEK IV & A. Phillips \\
\hline 1990 Nov 13 & $1.5-\mathrm{m}$ & CTIO & TEK IV & A. Walker \\
\hline 1990 Nov 21 & $0.91-\mathrm{m}$ & CTIO & Т13 & B. Chaboyer \\
\hline 1990 Nov 22 & $0.91-\mathrm{m}$ & CTIO & TI3 & B. Chaboyer \\
\hline 1990 Nov 23 & $0.91-\mathrm{m}$ & CTIO & TI3 & M. Navarrete \\
\hline 1990 Dec 14 & $0.91-\mathrm{m}$ & CTIO & THOM2 & M. Navarrete \\
\hline 1991 Apr 07 & $0.91-\mathrm{m}$ & CTIO & TEK2 & P. Ugarte \\
\hline 1991 Apr 09 & $4.0-\mathrm{m}$ & CTIO & TEK1 & M. Navarrete \\
\hline 1991 Apr 13 & $1.0-\mathrm{m}$ & LCO & FORD & D. Tucker \\
\hline 1991 Apr 14 & $1.0-\mathrm{m}$ & $\mathrm{LCO}$ & FORD & D. Tucker \\
\hline 1991 Apr 16 & $1.0-\mathrm{m}$ & $\mathrm{LCO}$ & FORD & D. Tucker \\
\hline 1991 Apr 17 & $0.91-\mathrm{m}$ & CTIO & TEK IV & R. Guhathakurta \\
\hline 1991 Apr 18 & $0.91-\mathrm{m}$ & CTIO & TEK IV & R. Guhathakurta \\
\hline 1991 Apr 19 & $0.91-\mathrm{m}$ & CTIO & TI3 & R. Schommer \\
\hline 1991 Apr 20 & $0.91-\mathrm{m}$ & CTIO & TI3 & L. González/J. Baldwin \\
\hline 1991 Apr 20 & $1.0-\mathrm{m}$ & $\mathrm{LCO}$ & FORD & D. Tucker \\
\hline 1991 Apr 24 & $0.91-\mathrm{m}$ & CTIO & TI3 & C. Bailyn \\
\hline 1991 Apr 26 & $0.91-\mathrm{m}$ & CTIO & TEK1 & R. Schommer \\
\hline 1991 Apr 27 & $0.91-\mathrm{m}$ & CTIO & TEK1 & R. Schommer \\
\hline 1991 Apr 28 & $0.91-\mathrm{m}$ & CTIO & TI3 & L. Wells \\
\hline 1991 Apr 29 & $0.91-\mathrm{m}$ & CTIO & TI3 & L. Wells \\
\hline 1991 May 01 & $0.91-\mathrm{m}$ & CTIO & TEK IV & N. Saavedra \\
\hline 1991 May 04 & $0.91-\mathrm{m}$ & CTIO & TEK IV & G. Williger \\
\hline 1991 May 05 & $0.91-\mathrm{m}$ & CTIO & TEK IV & G. Williger \\
\hline 1991 May 07 & $0.91-\mathrm{m}$ & CTIO & TEK IV & M. Navarrete \\
\hline 1991 May 09 & $4.0-\mathrm{m}$ & CTIO & TEK1 & M. Navarrete \\
\hline 1991 May 10 & $0.91-\mathrm{m}$ & CTIO & TI3 & N. Tyson \\
\hline 1991 May 17 & $0.91-\mathrm{m}$ & CTIO & TI3 & L. González \\
\hline 1991 May 29 & $0.91-\mathrm{m}$ & CTIO & TEK2 & G. Williger \\
\hline 1991 Jun 07 & $4.0-\mathrm{m}$ & CTIO & TI2 & M. Navarrete \\
\hline 1991 Jun 12 & $0.91-\mathrm{m}$ & CTIO & TEK2 & A. Sarajedini \\
\hline 1991 Jun 13 & $0.91-\mathrm{m}$ & CTIO & TEK IV & M. Navarrete \\
\hline
\end{tabular}


TABLE 2. (continued)

\begin{tabular}{|c|c|c|c|c|}
\hline Date (UT) & Telescope & Observatory & $\mathrm{CCD}$ & Observer(s) \\
\hline 1991 Jun 21 & $0.91-\mathrm{m}$ & CTIO & TEK IV & M. Shara \\
\hline 1991 Jun 29 & 0.91-m & CTIO & TEK1 & P. Seitzer \\
\hline 1991 Jul 01 & 0.91-m & CTIO & TEK1 & P. Seitzer \\
\hline 1991 Jul 02 & $0.91-\mathrm{m}$ & CTIO & $\mathrm{TI} 3$ & R. Schommer \\
\hline $1991 \mathrm{Jul} 03$ & $0.91-\mathrm{m}$ & CTIO & $\mathrm{TI} 3$ & R. Schommer \\
\hline $1991 \mathrm{Jul} 04$ & $0.91-\mathrm{m}$ & CTIO & TI3 & J. Baldwin \\
\hline 1991 Jul 05 & $0.91-\mathrm{m}$ & CTIO & $\mathrm{TI} 3$ & M. Navarrete \\
\hline 1991 Jul 06 & $0.91-\mathrm{m}$ & CTIO & TEK2 & L. Siciliano \\
\hline 1991 Jul 12 & $0.91-\mathrm{m}$ & CTIO & TEK2 & L. Siciliano \\
\hline 1991 Jul 21 & $0.91-\mathrm{m}$ & CTIO & TEK2 & A. Dey \\
\hline 1991 Aug 10 & $4.0-\mathrm{m}$ & CTIO & TEK IV & M. Navarrete \\
\hline 1991 Aug 13 & $1.5-\mathrm{m}$ & CTIO & TEK1 & M. T. Ruiz \\
\hline 1991 Aug 27 & $0.91-\mathrm{m}$ & CTIO & TEK2 & G. Williger \\
\hline 1991 Sep 06 & $4.0-\mathrm{m}$ & CTIO & TEK1 & M. Navarrete \\
\hline 1991 Sep 09 & 0.91-m & CTIO & TEK2 & N. Suntzeff \\
\hline 1991 Oct 08 & $0.91-\mathrm{m}$ & CTIO & TI3 & N. Suntzeff \\
\hline 1991 Oct 22 & $0.91-\mathrm{m}$ & CTIO & TEK1 & M. Hamuy \\
\hline 1991 Oct 23 & 0.91-m & CTIO & TEK1 & M. Hamuy \\
\hline 1991 Oct 24 & $0.91-\mathrm{m}$ & CTIO & TEK1 & M. Hamuy \\
\hline 1991 Oct 25 & $0.91-\mathrm{m}$ & CTIO & TEK1 & M. Hamuy \\
\hline 1991 Oct 26 & $0.91-\mathrm{m}$ & CTIO & TEK1 & M. Hamuy \\
\hline 1991 Oct 30 & $0.91-\mathrm{m}$ & CTIO & TEK1 & G. Williger \\
\hline 1991 Nov 11 & $0.91-\mathrm{m}$ & CTIO & TI3 & H. Ferguson \\
\hline 1992 Feb 26 & $0.91-\mathrm{m}$ & CTIO & TEK1 & N. Suntzeff \\
\hline 1992 Mar 05 & $0.91-\mathrm{m}$ & CTIO & TEK2 & K. Janes \\
\hline 1992 Mar 06 & $4.0-\mathrm{m}$ & CTIO & TEK2 & N. Suntzeff \\
\hline 1992 Mar 08 & $0.91-\mathrm{m}$ & CTIO & TEK1 & K. Janes \\
\hline 1992 Mar 10 & $0.91-\mathrm{m}$ & CTIO & TEK IV & M. Hamuy \\
\hline 1992 Mar 10 & NTT & ESO & THOM18 & J. Danziger \\
\hline 1992 Mar 12 & $0.91-\mathrm{m}$ & CTIO & TEK1 & C. Prosser \\
\hline 1992 Mar 13 & $0.91-\mathrm{m}$ & CTIO & TEK2 & C. Prosser \\
\hline 1992 Mar 19 & $0.91-\mathrm{m}$ & CTIO & TEK IV & P. Hartigan/J. Hughes \\
\hline 1992 Mar 19 & $1.0-\mathrm{m}$ & $\mathrm{LCO}$ & TEK & W. Krzeminski \\
\hline 1992 Mar 29 & $1.5-\mathrm{m}$ & CTIO & TEK2 & M. T. Ruiz \\
\hline 1992 Apr 04 & $0.91-\mathrm{m}$ & CTIO & TEK2 & C. Bailyn/Y-C. Kim \\
\hline 1992 Apr 05 & $0.91-\mathrm{m}$ & CTIO & TEK2 & C. Bailyn/Y-C. Kim \\
\hline 192 Apr 06 & $0.91-\mathrm{m}$ & CTIO & TEK2 & C. Bailyn/Y-C. Kim \\
\hline 1992 Apr 07 & $0.91-\mathrm{m}$ & CTIO & TEK2 & C. Bailyn/Y-C. Kim \\
\hline 1992 Apr 07 & $2.2-\mathrm{m}$ & ESO & THOM19 & M. Della Valle \\
\hline 1992 Apr 08 & $0.91-\mathrm{m}$ & CTIO & TEK2 & Y-C. Kim \\
\hline 1992 Apr 09 & $0.91-\mathrm{m}$ & CTIO & TEK2 & Y-C. Kim \\
\hline 1992 Apr 12 & $0.91-\mathrm{m}$ & CTIO & TEK2 & Y-C. Kim \\
\hline 1992 Apr 14 & $0.91-\mathrm{m}$ & CTIO & TEK2 & Y-C. Kim \\
\hline 1992 Apr 16 & $0.91-\mathrm{m}$ & CTIO & TEK1 & L. Wells \\
\hline 1992 Apr 19 & 0.91-m CTIO & TEK1 & R. Schommer & \\
\hline 1992 Apr 20 & $0.91-\mathrm{m}$ & CTIO & TEK1 & R. Schommer \\
\hline 1992 May 11 & NTT & ESO & THOM18 & M. Della Valle \\
\hline 1992 May 20 & 0.91-m & CTIO & TEK2 & G. Williger \\
\hline 1992 May 25 & 0.91-m & CTIO & TEK1 & M. Hamuy/R. Avilés \\
\hline 1992 Jun 03 & $0.91-\mathrm{m}$ & CTIO & TEK IV & L. Wells \\
\hline 1992 Jun 20 & $0.91-\mathrm{m}$ & CTIO & TEK1 & E. Rubenstein \\
\hline 1992 Jun 27 & $0.91-\mathrm{m}$ & CTIO & TEK1 & S. Cersosimo \\
\hline 1992 Jul 02 & $0.91-\mathrm{m}$ & CTIO & TEK4 & K. Wakamatsu/M. Malkan \\
\hline 1992 Jul 02 & $1.5-\mathrm{m}$ & CTIO & $\cdots$ & C. Anguita \\
\hline 1992 Jul 03 & $0.91-\mathrm{m}$ & CTIO & TEK1 & K. Wakamatsu/M. Malkan \\
\hline 1992 Jul 03 & $3.6-\mathrm{m}$ & ESO & TEK26 & M. Della Valle \\
\hline 1992 Jul 04 & $0.91-\mathrm{m}$ & CTIO & TEK1 & M. Hamuy/R. Avilés \\
\hline 1992 Jul 05 & $0.91-\mathrm{m}$ & CTIO & TEK3 & J. Baldwin \\
\hline 1992 Jul 06 & 0.91-m & CTIO & TEK3 & A. Walker \\
\hline 1992 Jul 09 & $0.91-\mathrm{m}$ & CTIO & TEK3 & A. Walker \\
\hline 1992 Jul 10 & $0.91-\mathrm{m}$ & CTIO & TEK3 & A. Walker \\
\hline 1992 Jul 12 & $1.0-\mathrm{m}$ & $\mathrm{LCO}$ & FORD2 & M. Hamuy/R. Avilés \\
\hline 1992 Jul 13 & $1.0-\mathrm{m}$ & $\mathrm{LCO}$ & FORD2 & M. Hamuy/R. Avilés \\
\hline 1992 Jul 15 & $1.0-\mathrm{m}$ & $\mathrm{LCO}$ & FORD2 & R. Avilés/F. Barrientos \\
\hline 1992 Jul 15 & $1.0-\mathrm{m}$ & $\mathrm{LCO}$ & FORD2 & R. Avilés/F. Barrientos \\
\hline 1992 Jul 19 & $0.91-\mathrm{m}$ & CTIO & TEK1 & E. Costa \\
\hline 1992 Jul 22 & $0.91-\mathrm{m}$ & CTIO & TEK1 & E. Costa \\
\hline
\end{tabular}


TABLE 2. (continued)

\begin{tabular}{|c|c|c|c|c|}
\hline Date (UT) & Telescope & Observatory & $\mathrm{CCD}$ & Observer(s) \\
\hline 1992 Jul 27 & $0.91-\mathrm{m}$ & CTIO & TEK3 & N. Tyson/R. Gal \\
\hline 1992 Jul 28 & $0.91-\mathrm{m}$ & CTIO & TEK3 & N. Tyson/R. Gal \\
\hline 1992 Jul 29 & $0.91-\mathrm{m}$ & CTIO & TEK3 & N. Tyson/R. Gal \\
\hline 1992 Jul 29 & $0.91-\mathrm{m}$ & CTIO & TEK3 & N. Tyson/R. Gal \\
\hline 1992 Jul 31 & $0.91-\mathrm{m}$ & CTIO & TEK3 & N. Tyson/R. Gal \\
\hline 1992 Aug 01 & $2.2-\mathrm{m}$ & ESO & THOM19 & M. Della Valle \\
\hline 1992 Aug 01 & $0.91-\mathrm{m}$ & CTIO & TEK1 & R. Avilés/C. Smith \\
\hline 1992 Aug 02 & $0.91-\mathrm{m}$ & CTIO & TEK1 & R. Avilés/C. Smith \\
\hline 1992 Aug 03 & $0.91-\mathrm{m}$ & CTIO & TEK1 & E. Rubenstein \\
\hline 1992 Aug 06 & $0.91-\mathrm{m}$ & CTIO & TEK1 & E. Rubenstein \\
\hline 1992 Aug 08 & $0.91-\mathrm{m}$ & CTIO & TEK1 & R. Avilés \\
\hline 1992 Aug 09 & $0.91-\mathrm{m}$ & CTIO & TEK1 & R. Avilés \\
\hline 1992 Aug 10 & $0.91-\mathrm{m}$ & CTIO & TEK1 & R. Avilés \\
\hline 1992 Aug 11 & $0.91-\mathrm{m}$ & CTIO & TEK1 & R. Avilés \\
\hline 1992 Aug 12 & $0.91-\mathrm{m}$ & CTIO & TEK1 & R. Avilés/M. Hamuy \\
\hline 1992 Aug 13 & $0.91-\mathrm{m}$ & CTIO & TEK1 & R. Avilés \\
\hline 1992 Aug 14 & $0.91-\mathrm{m}$ & CTIO & TEK1 & R. Avilés \\
\hline 1992 Aug 15 & $0.91-\mathrm{m}$ & CTIO & TEK1 & R. Avilés/F. Barrientos \\
\hline 1992 Aug 20 & $0.91-\mathrm{m}$ & CTIO & TEK1 & S. Cersosimo \\
\hline 1992 Aug 21 & $0.91-\mathrm{m}$ & CTIO & TEK1 & S. Cersosimo \\
\hline 1992 Sep 03 & $0.91-\mathrm{m}$ & CTIO & TEK1 & R. Avilés \\
\hline 1992 Sep 05 & $0.91-\mathrm{m}$ & CTIO & TEK1 & R. Avilés \\
\hline $1992 \mathrm{Sep} 06$ & $0.91-\mathrm{m}$ & CTIO & TEK1 & R. Avilés \\
\hline 1992 Sep 07 & $0.91-\mathrm{m}$ & CTIO & TEK1 & R. Avilés \\
\hline 1992 Sep 20 & $0.91-\mathrm{m}$ & CTIO & TEK1 & R. Avilés \\
\hline 1992 Sep 26 & $0.91-\mathrm{m}$ & CTIO & TEK1 & R. Avilés \\
\hline 1992 Oct 06 & $0.91-\mathrm{m}$ & CTIO & TEK3 & R. McMahan/G. Baggley \\
\hline 1992 Oct 07 & $0.91-\mathrm{m}$ & CTIO & TEK3 & R. McMahan/G. Baggley \\
\hline 1992 Oct 08 & $0.91-\mathrm{m}$ & CTIO & TEK3 & R. McMahan/G. Baggley \\
\hline 1992 Oct 09 & $0.91-\mathrm{m}$ & CTIO & TEK1 & R. Avilés/M. Hamuy \\
\hline 1992 Oct 10 & $0.91-\mathrm{m}$ & CTIO & TEK1 & R. Avilés/M. Hamuy \\
\hline 1992 Oct 11 & $0.91-\mathrm{m}$ & CTIO & TEK3 & C. Smith \\
\hline 1992 Oct 12 & $0.91-\mathrm{m}$ & CTIO & TEK3 & C. Smith \\
\hline 1992 Oct 13 & $0.91-\mathrm{m}$ & CTIO & TEK1 & R. Avilés \\
\hline 1992 Oct 17 & $0.91-\mathrm{m}$ & CTIO & TEK IV & E. Costa \\
\hline 1992 Oct 19 & $0.91-\mathrm{m}$ & CTIO & TEK IV & E. Costa \\
\hline 1992 Oct 20 & $0.91-\mathrm{m}$ & CTIO & TEK IV & E. Costa/M. Hamuy \\
\hline 1992 Oct 20 & $1.5-\mathrm{m}$ & CTIO & TI3 & F. Barrientos \\
\hline 1992 Oct 21 & $0.91-\mathrm{m}$ & CTIO & TEK IV & E. Costa \\
\hline 1992 Oct 24 & $4.0-\mathrm{m}$ & CTIO & TEK1 & M. Navarrete \\
\hline 1992 Oct 25 & $0.91-\mathrm{m}$ & CTIO & TEK4 & L. González/E. Costa \\
\hline 1992 Oct 27 & $0.91-\mathrm{m}$ & CTIO & TEK IV & R. Avilés \\
\hline 1992 Oct 28 & $0.91-\mathrm{m}$ & CTIO & TEK3 & N. Caldwell \\
\hline 1992 Oct 29 & $0.91-\mathrm{m}$ & CTIO & TEK3 & N. Caldwell \\
\hline 1992 Oct 30 & $0.91-\mathrm{m}$ & CTIO & TI3 & G. Williger \\
\hline 1992 Oct 31 & $0.91-\mathrm{m}$ & CTIO & TI3 & G. Williger \\
\hline 1992 Nov 08 & $0.91-\mathrm{m}$ & CTIO & TEK1 & R. Avilés \\
\hline 1992 Nov 14 & $0.91-\mathrm{m}$ & CTIO & TEK IV & R. Avilés \\
\hline 1992 Nov 15 & $0.91-\mathrm{m}$ & CTIO & TEK IV & R. Avilés \\
\hline 1992 Nov 20 & $0.91-\mathrm{m}$ & CTIO & TEK3 & R. Avilés \\
\hline 1992 Nov 21 & $0.91-\mathrm{m}$ & CTIO & TEK3 & K-P. Cheng \\
\hline 1992 Nov 23 & $0.91-\mathrm{m}$ & CTIO & TEK3 & K-P. Cheng \\
\hline 1992 Nov 24 & $0.91-\mathrm{m}$ & CTIO & TEK3 & K-P. Cheng \\
\hline 1992 Nov 27 & $0.91-\mathrm{m}$ & CTIO & TI3 & A. Layden \\
\hline 1992 Nov 28 & $0.91-\mathrm{m}$ & CTIO & TI3 & J. Elias/F. Barrientos \\
\hline 1992 Nov 29 & $0.91-\mathrm{m}$ & CTIO & TI3 & J. Elias/F. Barrientos \\
\hline 1992 Nov 30 & $0.91-\mathrm{m}$ & CTIO & TI3 & J. Elias/F. Barrientos \\
\hline 1992 Dec 05 & $0.91-\mathrm{m}$ & CTIO & TEK3 & R. Schommer \\
\hline 1992 Dec 07 & $0.91-\mathrm{m}$ & CTIO & TEK1 & R. Avilés \\
\hline $1992 \operatorname{Dec} 13$ & $0.91-\mathrm{m}$ & CTIO & TEK IV & R. Avilés \\
\hline 1992 Dec 19 & $0.91-\mathrm{m}$ & CTIO & TEK IV & R. Avilés \\
\hline 1992 Dec 22 & $0.91-\mathrm{m}$ & CTIO & TEK IV & R. Avilés \\
\hline 1992 Dec 23 & $0.91-\mathrm{m}$ & CTIO & TEK IV & R. Avilés \\
\hline $1992 \operatorname{Dec} 23$ & $4.0-\mathrm{m}$ & CTIO & TEK1 & N. Suntzeff \\
\hline 1992 Dec 24 & $0.91-\mathrm{m}$ & CTIO & TEK IV & R. Schommer \\
\hline $1992 \operatorname{Dec} 26$ & $0.91-\mathrm{m}$ & CTIO & TEK3 & N. Caldwell \\
\hline 1992 Dec 30 & $0.91-\mathrm{m}$ & CTIO & TEK IV & R. Avilés \\
\hline
\end{tabular}


TABLE 2. (continued)

\begin{tabular}{|c|c|c|c|c|}
\hline Date (UT) & Telescope & Observatory & $\mathrm{CCD}$ & Observer(s) \\
\hline 1992 Dec 31 & $0.91-\mathrm{m}$ & CTIO & TEK IV & R. Avilés \\
\hline 1993 Jan 03 & $4.0-\mathrm{m}$ & CTIO & TEK1 & N. Caldwell \\
\hline 1993 Jan 03 & $0.91-\mathrm{m}$ & CTIO & TEK3 & R. Méndez \\
\hline $1993 \operatorname{Jan} 04$ & $0.91-\mathrm{m}$ & CTIO & TEK3 & R. Méndez \\
\hline 1993 Jan 07 & $0.91-\mathrm{m}$ & CTIO & TEK1 & R. Avilés \\
\hline 1993 Jan 10 & $0.91-\mathrm{m}$ & CTIO & TEK1 & R. Avilés \\
\hline 1993 Jan 11 & $0.91-\mathrm{m}$ & CTIO & TEK1 & R. Avilés \\
\hline 1993 Jan 12 & $0.91-\mathrm{m}$ & CTIO & TEK1 & R. Avilés \\
\hline 1993 Jan 20 & $1.5-\mathrm{m}$ & CTIO & TEK1 & C. Anguita \\
\hline 1993 Jan 24 & $0.91-\mathrm{m}$ & CTIO & TEK IV & R. Avilés \\
\hline $1993 \operatorname{Jan} 25$ & $0.91-\mathrm{m}$ & CTIO & TEK IV & R. Avilés \\
\hline 1993 Jan 26 & $0.91-\mathrm{m}$ & CTIO & TEK IV & R. Avilés \\
\hline $1993 \operatorname{Jan} 29$ & $0.91-\mathrm{m}$ & CTIO & TEK IV & R. Avilés \\
\hline $1993 \operatorname{Jan} 30$ & $0.91-\mathrm{m}$ & CTIO & TEK IV & R. Avilés \\
\hline 1993 Feb 08 & $0.91-\mathrm{m}$ & CTIO & TEK3 & M. Hamuy/X. Gómez \\
\hline 1993 Feb 17 & $4.0-\mathrm{m}$ & CTIO & TEK1 & R. Schommer \\
\hline 1993 Feb 19 & $0.91-\mathrm{m}$ & CTIO & TEK IV & R. Avilés \\
\hline 1993 Feb 20 & $0.91-\mathrm{m}$ & CTIO & TEK IV & R. Avilés \\
\hline 1993 Feb 22 & $4.0-\mathrm{m}$ & CTIO & TEK1 & N. Suntzeff \\
\hline 1993 Mar 05 & $0.91-\mathrm{m}$ & CTIO & TEK1 & R. Koopmann \\
\hline 1993 Mar 07 & $0.91-\mathrm{m}$ & CTIO & TEK1 & R. Avilés \\
\hline 1993 Mar 17 & $0.91-\mathrm{m}$ & CTIO & TEK3 & R. Avilés \\
\hline 1993 Mar 21 & $0.91-\mathrm{m}$ & CTIO & TEK3 & D. Geisler \\
\hline $1993 \operatorname{Mar} 22$ & $0.91-\mathrm{m}$ & CTIO & TEK3 & D. Geisler \\
\hline 1993 Mar 23 & $0.91-\mathrm{m}$ & CTIO & TI3 & J. Baldwin \\
\hline 1993 Mar 24 & $0.91-\mathrm{m}$ & CTIO & TEK3 & R. Havlen \\
\hline 1993 Mar 25 & $0.91-\mathrm{m}$ & CTIO & TEK3 & R. Havlen \\
\hline 1993 Mar 26 & $0.91-\mathrm{m}$ & CTIO & TEK3 & R. Havlen \\
\hline 1993 Mar 27 & $0.91-\mathrm{m}$ & CTIO & TEK3 & R. Avilés \\
\hline 1993 Apr 01 & $0.91-\mathrm{m}$ & CTIO & TEK1 & R. Avilés \\
\hline 1993 Apr 06 & $0.91-\mathrm{m}$ & CTIO & TEK3 & C. Prosser \\
\hline 1993 Apr 07 & $0.91-\mathrm{m}$ & CTIO & TEK1 & R. Avilés \\
\hline 1993 Apr 11 & $0.91-\mathrm{m}$ & CTIO & TEK1 & A. Layden \\
\hline 1993 Apr 13 & $0.91-\mathrm{m}$ & CTIO & TEK1 & A. Layden \\
\hline 1993 Apr 20 & $0.91-\mathrm{m}$ & CTIO & TEK3 & R. Avilés \\
\hline 1993 Apr 20 & $4.0-\mathrm{m}$ & CTIO & TEK2 & N. Suntzeff \\
\hline 1993 Apr 26 & $0.91-\mathrm{m}$ & CTIO & TEK1 & G. Williger \\
\hline 1993 May 01 & $0.91-\mathrm{m}$ & CTIO & TEK1 & G. Williger \\
\hline 1993 May 02 & $0.91-\mathrm{m}$ & CTIO & TEK1 & R. Avilés \\
\hline 1993 May 17 & $0.91-\mathrm{m}$ & CTIO & TEK3 & R. Avilés \\
\hline 1993 May 19 & $0.91-\mathrm{m}$ & CTIO & TEK3 & R. Avilés \\
\hline 1993 May 20 & $0.91-\mathrm{m}$ & CTIO & TEK3 & A. Layden \\
\hline 1993 May 22 & $0.91-\mathrm{m}$ & CTIO & TEK3 & A. Layden \\
\hline 1993 May 25 & $0.91-\mathrm{m}$ & CTIO & TEK3 & A. Walker \\
\hline 1993 May 26 & $0.91-\mathrm{m}$ & CTIO & TEK3 & A. Walker \\
\hline 1993 May 28 & $0.91-\mathrm{m}$ & CTIO & TEK1 & R. Avilés \\
\hline 1993 Jun 01 & $0.91-\mathrm{m}$ & CTIO & TEK1 & L. French \\
\hline 1993 Jun 04 & $0.91-\mathrm{m}$ & CTIO & TEK1 & R. Avilés \\
\hline 1993 Jun 05 & $0.91-\mathrm{m}$ & CTIO & TEK1 & R. Avilés \\
\hline 1993 Jun 08 & $0.91-\mathrm{m}$ & CTIO & TEK1 & M. Hernández \\
\hline 1993 Jun 09 & $0.91-\mathrm{m}$ & CTIO & TEK1 & R. Schommer \\
\hline 1993 Jun 10 & $0.91-\mathrm{m}$ & CTIO & TEK1 & R. Schommer \\
\hline 1993 Jun 13 & $4.0-\mathrm{m}$ & CTIO & TEK3 & A. Walker \\
\hline 1993 Jun 14 & $0.91-\mathrm{m}$ & CTIO & TEK1 & R. Avilés \\
\hline 1993 Jun 16 & $0.91-\mathrm{m}$ & CTIO & TEK1 & R. Avilés \\
\hline 1993 Jun 19 & $0.91-\mathrm{m}$ & CTIO & TEK1 & M. Bolte/J. Hesser \\
\hline 1993 Jun 23 & $0.91-\mathrm{m}$ & CTIO & TI3 & E. Rubenstein/W. Sherry \\
\hline 1993 Jun 24 & $4.0-\mathrm{m}$ & CTIO & TEK3 & N. Suntseff \\
\hline 1993 Jul 08 & $0.91-\mathrm{m}$ & CTIO & TEK1 & W. Sherry \\
\hline 1993 Jul 11 & $0.91-\mathrm{m}$ & CTIO & TEK1 & R. Avilés \\
\hline 1993 Jul 16 & $0.91-\mathrm{m}$ & CTIO & TEK2 & R. Avilés \\
\hline 1993 Jul 17 & $0.91-\mathrm{m}$ & CTIO & TEK2 & R. Avilés \\
\hline 1993 Jul 18 & $0.91-\mathrm{m}$ & CTIO & TEK2 & R. Avilés \\
\hline 1993 Jul 19 & $0.91-\mathrm{m}$ & CTIO & TEK2 & R. Avilés \\
\hline 1993 Aug 03 & $0.91-\mathrm{m}$ & CTIO & TEK2 & R. Avilés \\
\hline 1993 Aug 22 & $0.91-\mathrm{m}$ & CTIO & TEK2 & R. Avilés \\
\hline 1993 Sep 09 & $4.0-\mathrm{m}$ & CTIO & TEK3 & M. Navarrete \\
\hline
\end{tabular}




\begin{tabular}{|c|c|c|c|c|}
\hline Date (UT) & Telescope & Observatory & $\mathrm{CCD}$ & Observer(s) \\
\hline 1993 Sep 17 & $0.91-\mathrm{m}$ & CTIO & $\mathrm{TI} 3$ & P. Francis \\
\hline 1993 Sep 18 & $0.91-\mathrm{m}$ & CTIO & TEK2 & R. Avilés \\
\hline 1993 Nov 06 & $4.0-\mathrm{m}$ & CTIO & TEK3 & R. Avilés \\
\hline 1993 Nov 12 & $4.0-\mathrm{m}$ & CTIO & $\cdots$ & J. Maza/P. Ortiz \\
\hline 1993 Nov 16 & $0.91-\mathrm{m}$ & CTIO & TEK2 & R. Avilés \\
\hline 1993 Nov 23 & $0.91-\mathrm{m}$ & CTIO & TEK3 & L. Ho \\
\hline 1993 Nov 24 & $0.91-\mathrm{m}$ & CTIO & TEK3 & L. Ho \\
\hline 1993 Nov 29 & $0.91-\mathrm{m}$ & CTIO & TEK3 & D. Welch \\
\hline 1993 Dec 01 & $0.91-\mathrm{m}$ & CTIO & TEK3 & A. Walker \\
\hline $1993 \mathrm{Dec} 03$ & $0.91-\mathrm{m}$ & CTIO & TEK2 & A. Layden \\
\hline 1993 Dec 05 & $0.91-\mathrm{m}$ & CTIO & TEK2 & A. Layden \\
\hline 1993 Dec 17 & $0.91-\mathrm{m}$ & CTIO & TEK2 & R. Avilés \\
\hline 1993 Dec 29 & $0.91-\mathrm{m}$ & CTIO & TEK2 & J. Storm \\
\hline 1993 Dec 30 & $0.91-\mathrm{m}$ & CTIO & TEK2 & J. Storm \\
\hline 1993 Dec 31 & $0.91-\mathrm{m}$ & CTIO & TEK2 & J. Storm \\
\hline 1994 Jan 01 & $0.91-\mathrm{m}$ & CTIO & TEK2 & J. Storm \\
\hline 1994 Jan 02 & $0.91-\mathrm{m}$ & CTIO & TEK2 & J. Storm \\
\hline 1994 Jan 02 & $4.0-\mathrm{m}$ & CTIO & TEK3 & R. Elston \\
\hline 1994 Jan 03 & $0.91-\mathrm{m}$ & CTIO & TEK2 & J. Storm \\
\hline 1994 Jan 14 & $0.91-\mathrm{m}$ & CTIO & TEK2 & R. Avilés/P. Lira \\
\hline $1994 \operatorname{Jan} 22$ & $0.91-\mathrm{m}$ & CTIO & TEK2 & R. Avilés \\
\hline 1994 Feb 05 & $0.91-\mathrm{m}$ & CTIO & TEK2 & R. Avilés/P. Lira \\
\hline 1994 Feb 06 & $0.91-\mathrm{m}$ & CTIO & TEK2 & R. Avilés/P. Lira \\
\hline 1994 Mar 14 & $0.91-\mathrm{m}$ & CTIO & TEK2 & R. Avilés/P. Lira \\
\hline 1994 Mar 17 & $4.0-\mathrm{m}$ & CTIO & TEK3 & N. Suntzeff/M. Phillips \\
\hline 1994 May 10 & $0.91-\mathrm{m}$ & CTIO & TEK2 & R. Avilés \\
\hline 1994 May 11 & $0.91-\mathrm{m}$ & CTIO & TEK2 & R. Avilés \\
\hline 1994 Jun 06 & $0.91-\mathrm{m}$ & CTIO & TEK2 & M. Hernández \\
\hline 1994 Jul 12 & $4.0-\mathrm{m}$ & CTIO & TEK4 & R. Avilés \\
\hline 1994 Jul 31 & $0.91-\mathrm{m}$ & CTIO & TEK3 & R. Avilés \\
\hline 1994 Sep 12 & $4.0-\mathrm{m}$ & CTIO & TEK4 & R. Avilés \\
\hline 1994 Nov 09 & $4.0-\mathrm{m}$ & CTIO & TEK4 & N. Suntzeff \\
\hline 1995 Feb 10 & $0.91-\mathrm{m}$ & CTIO & TEK2 & R. Avilés \\
\hline 1995 Feb 11 & $0.91-\mathrm{m}$ & CTIO & TEK2 & R. Avilés \\
\hline
\end{tabular}

tem, and also due to the subtraction of the underlying host galaxy.

\section{MAXIMUM-LIGHT MAGNITUDES \& DECLINE RATES}

Figure 3 shows the $B V I$ light curves of the $29 \mathrm{SNe}$ Ia included in this study. Maximum-light magnitudes were derived for each $\mathrm{SN}$ in one of the following two methods:

(1) Direct Measurement. For 11 SNe (slightly more than one third of the sample), photometry was obtained at or before maximum light allowing direct measurement of the maximum-light magnitudes in $B$ and $V$. However, for several of these objects (e.g., see the light curves of SN 1992ag in Fig. 3), coverage of the $I$ light curve was insufficient to allow direct measurement of the maximum-light brightness in this band. In these cases, the best-fitting template (see below) was used, often adjusting this to the first $I$ data point. The corresponding error in the peak magnitude was taken to be $0.03^{m}$ in those cases where the coverage of the light curve started before maximum, and $0.05^{m}$ when the observations started only one or two days before the peak.

(2) Template Fitting. For the majority of the $\mathrm{SNe}$ in our sample, the light curve observations did not begin until after maximum light. To estimate peak magnitudes for these events, we employed a template fitting procedure similar to that utilized in Paper III and Hamuy et al. 1995 (hereafter referred to as Paper IV). As detailed in a separate paper (Hamuy et al. 1996c; hereafter referred to as Paper VIII), a family of six $B V I$ light curve templates, representing the range of observed decline rates of $\mathrm{SNe} \mathrm{Ia}$, were produced from precise CCD photometry obtained at CTIO of seven well-observed events (1992bc, 1991T, 1992al, 1992A, $1992 \mathrm{bo}, 1993 \mathrm{H}$, and $1991 \mathrm{bg}$ ). These templates were fit to the observed photometry of each of the program $\mathrm{SNe}$ via a $\chi^{2}$-minimizing technique which solved simultaneously for the time of $B$ maximum and the peak magnitudes $B_{M A X}$, $V_{M A X}$, and $I_{M A X}$. (Note that in our previous papers, the $I$-band data was not included.) As detailed in Paper III, before performing these fits, the templates were first modified by the appropriate $K$ terms (Hamuy et al. 1993b) and were also stretched to account for time dilation. For about half of the $\mathrm{SNe}$, one of the templates provided a much better fit (as judged by the value of the reduced $\chi^{2}$ ) than the others. An example is SN 1992ae (see Fig. 3) whose BVI light curves were found to be an excellent match to the SN 1992al templates. However, for many of the program $\mathrm{SNe}$, the data were fit essentially equally well by two different templates. A good example of such an event is SN 1991ag (see Fig. 3), for which the $1991 \mathrm{~T}$ and $1992 \mathrm{bc}$ templates yielded similar values of the reduced $\chi^{2}$. Hence, we adopted the general rule that when the difference in the reduced $\chi^{2}$ of two template fits was $\leqslant 1.5$, the peak magnitudes were obtained by aver- 
TABLE 3. $B V(R I)_{K C}$ sequences.

\begin{tabular}{|c|c|c|c|c|c|c|c|c|}
\hline Star & $B$ & $\sigma_{B}$ & $V$ & $\sigma_{V}$ & $R$ & $\sigma_{R}$ & $I$ & $\sigma_{I}$ \\
\hline \multicolumn{9}{|c|}{ SN 19900} \\
\hline $\mathrm{c} 1$ & 18.750 & 0.029 & 17.897 & 0.011 & 17.407 & 0.007 & 16.940 & 0.009 \\
\hline $\mathrm{c} 2$ & 19.556 & 0.059 & 18.395 & 0.027 & 17.622 & 0.018 & 16.905 & 0.021 \\
\hline c3 & 19.900 & 0.078 & 18.333 & 0.021 & 17.401 & 0.010 & 16.569 & 0.011 \\
\hline $\mathrm{c} 4$ & 19.178 & 0.038 & 18.103 & 0.014 & 17.434 & 0.008 & 16.878 & 0.011 \\
\hline$c 5$ & 18.796 & 0.030 & 18.112 & 0.013 & 17.701 & 0.008 & 17.312 & 0.011 \\
\hline$c 6$ & 19.193 & 0.040 & 18.414 & 0.017 & 17.994 & 0.012 & 17.605 & 0.022 \\
\hline c7 & 18.588 & 0.024 & 17.760 & 0.011 & 17.287 & 0.008 & 16.920 & 0.012 \\
\hline $\mathrm{c} 8$ & 18.295 & 0.019 & 17.434 & 0.008 & 16.916 & 0.005 & 16.450 & 0.008 \\
\hline $\mathrm{c} 10$ & 19.619 & 0.060 & 18.889 & 0.027 & 18.478 & 0.020 & 18.050 & 0.033 \\
\hline $\mathrm{c} 11$ & 18.387 & 0.020 & 17.415 & 0.008 & 16.811 & 0.004 & 16.281 & 0.006 \\
\hline $\mathrm{c} 13$ & 14.637 & 0.002 & 13.596 & 0.001 & $\ldots$ & $\cdots$ & $\ldots$ & $\ldots$ \\
\hline \multicolumn{9}{|c|}{ SN 1990T } \\
\hline $\mathrm{c} 1$ & 17.931 & 0.007 & 17.374 & 0.005 & 17.041 & 0.006 & 16.712 & 0.004 \\
\hline $\mathrm{c} 2$ & 19.742 & 0.023 & 18.561 & 0.004 & 17.846 & 0.004 & 17.208 & 0.004 \\
\hline c3 & 18.825 & 0.007 & 18.335 & 0.007 & 18.007 & 0.007 & 17.684 & 0.014 \\
\hline $\mathrm{c} 4$ & 19.577 & 0.013 & 19.018 & 0.007 & 18.705 & 0.007 & 18.322 & 0.016 \\
\hline $\mathrm{c} 5$ & 17.286 & 0.007 & 16.360 & 0.007 & 15.738 & 0.006 & 15.206 & 0.005 \\
\hline c6 & 15.425 & 0.006 & 14.535 & 0.004 & 14.043 & 0.004 & 13.609 & 0.004 \\
\hline c7 & 16.293 & 0.007 & 15.307 & 0.003 & 14.759 & 0.004 & 14.278 & 0.002 \\
\hline c8 & 17.303 & 0.005 & 16.691 & 0.004 & 16.328 & 0.003 & 15.968 & 0.004 \\
\hline \multicolumn{9}{|c|}{ SN 1990Y } \\
\hline $\mathrm{c} 2$ & 18.422 & 0.008 & 17.514 & 0.003 & 16.993 & 0.002 & 16.548 & 0.005 \\
\hline c3 & 20.214 & 0.031 & 18.636 & 0.007 & 17.540 & 0.003 & 16.236 & 0.004 \\
\hline c4 & 18.197 & 0.006 & 17.514 & 0.003 & 17.119 & 0.003 & 16.736 & 0.003 \\
\hline $\mathrm{c} 5$ & 20.034 & 0.024 & 18.595 & 0.007 & 17.630 & 0.002 & 16.547 & 0.003 \\
\hline c6 & 15.617 & 0.004 & 15.137 & 0.002 & 14.847 & 0.002 & 14.572 & 0.002 \\
\hline c7 & 17.484 & 0.005 & 16.710 & 0.002 & 16.285 & 0.003 & 15.896 & 0.002 \\
\hline $\mathrm{c} 8$ & 20.057 & 0.021 & 18.556 & 0.009 & 17.867 & 0.007 & 17.203 & 0.014 \\
\hline c9 & 16.553 & 0.006 & 15.904 & 0.003 & 15.535 & 0.002 & 15.181 & 0.002 \\
\hline $\mathrm{c} 10$ & 19.367 & 0.010 & 18.875 & 0.006 & 18.557 & 0.003 & 18.219 & 0.016 \\
\hline \multicolumn{9}{|c|}{ SN 1990af } \\
\hline $\mathrm{c} 1$ & 18.405 & 0.002 & 17.440 & 0.002 & 16.833 & 0.005 & 16.311 & 0.008 \\
\hline $\mathrm{c} 2$ & 20.110 & 0.005 & 19.629 & 0.006 & 19.346 & 0.047 & 19.086 & 0.091 \\
\hline c3 & 17.041 & 0.002 & 16.377 & 0.005 & 15.978 & 0.003 & 15.588 & 0.004 \\
\hline $\mathrm{c} 4$ & 16.270 & 0.007 & 15.670 & 0.005 & 15.284 & 0.002 & 14.918 & 0.003 \\
\hline $\mathrm{c5}$ & 18.604 & 0.002 & 17.853 & 0.005 & 17.417 & 0.008 & 17.010 & 0.014 \\
\hline c6 & $\cdots$ & $\cdots$ & 14.597 & 0.005 & 14.200 & 0.001 & 13.836 & 0.001 \\
\hline$c 8$ & 16.354 & 0.001 & 15.607 & 0.007 & 15.172 & 0.001 & 14.782 & 0.002 \\
\hline c9 & 17.902 & 0.002 & 16.528 & 0.005 & 15.619 & 0.002 & 14.911 & 0.002 \\
\hline $\mathrm{c} 10$ & 17.211 & 0.005 & 16.638 & 0.009 & $\ldots$ & $\cdots$ & $\cdots$ & $\ldots$ \\
\hline $\mathrm{c} 12$ & 17.362 & 0.012 & 16.156 & 0.010 & $\ldots$ & $\cdots$ & $\cdots$ & $\cdots$ \\
\hline $\mathrm{c} 13$ & 20.510 & 0.011 & 19.182 & 0.006 & $\ldots$ & $\ldots$ & $\cdots$ & $\ldots$ \\
\hline c14 & 20.556 & 0.011 & 19.055 & 0.002 & $\ldots$ & $\ldots$ & $\cdots$ & $\ldots$ \\
\hline \multicolumn{9}{|c|}{ SN 1991S } \\
\hline $\mathrm{c} 1$ & 18.502 & 0.020 & 18.029 & 0.008 & 17.703 & 0.007 & 17.398 & 0.007 \\
\hline $\mathrm{c} 2$ & 20.387 & 0.013 & 18.928 & 0.011 & 18.009 & 0.009 & 17.115 & 0.015 \\
\hline c3 & 18.271 & 0.017 & 17.583 & 0.007 & 17.179 & 0.008 & 16.789 & 0.006 \\
\hline $\mathrm{c} 4$ & 18.948 & 0.009 & 18.596 & 0.015 & 18.366 & 0.007 & 18.162 & 0.018 \\
\hline$c 5$ & 19.560 & 0.029 & 19.103 & 0.012 & 18.810 & 0.015 & 18.487 & 0.026 \\
\hline \multicolumn{9}{|c|}{ SN 1991U } \\
\hline $\mathrm{cl}$ & 15.966 & 0.004 & 15.015 & 0.002 & 14.439 & 0.002 & 13.906 & 0.013 \\
\hline$c 2$ & 17.709 & 0.007 & 16.828 & 0.009 & 16.338 & 0.007 & 15.906 & 0.008 \\
\hline c3 & 18.756 & 0.038 & 17.888 & 0.009 & 17.372 & 0.007 & 16.894 & 0.009 \\
\hline$c 4$ & 19.954 & 0.003 & 18.515 & 0.004 & 17.625 & 0.004 & 16.793 & 0.014 \\
\hline$c 5$ & 17.987 & 0.002 & 17.208 & 0.002 & 16.764 & 0.002 & 16.340 & 0.008 \\
\hline c6 & 18.851 & 0.008 & 17.703 & 0.002 & 16.984 & 0.002 & 16.363 & 0.007 \\
\hline c7 & 17.402 & 0.002 & 16.630 & 0.005 & 16.171 & 0.002 & 15.714 & 0.008 \\
\hline c8 & 18.026 & 0.005 & 17.288 & 0.003 & 16.854 & 0.002 & 16.427 & 0.005 \\
\hline c9 & 18.011 & 0.011 & 17.321 & 0.002 & 16.901 & 0.004 & 16.507 & 0.008 \\
\hline \multicolumn{9}{|c|}{ SN 1991ag } \\
\hline $\mathrm{cl}$ & 13.516 & 0.003 & 12.920 & 0.002 & 12.552 & 0.010 & 12.194 & 0.008 \\
\hline $\mathrm{c} 2$ & 16.472 & 0.004 & 15.619 & 0.007 & 15.117 & 0.003 & 14.679 & 0.008 \\
\hline c3 & 14.902 & 0.001 & 14.219 & 0.005 & 13.808 & 0.002 & 13.457 & 0.010 \\
\hline $\mathrm{c} 4$ & 17.394 & 0.003 & 16.749 & 0.006 & 16.359 & 0.002 & 15.988 & 0.008 \\
\hline$c 5$ & 17.185 & 0.008 & 16.444 & 0.005 & 16.011 & 0.006 & 15.603 & 0.008 \\
\hline
\end{tabular}


TABLE 3. (continued)

\begin{tabular}{|c|c|c|c|c|c|c|c|c|}
\hline Star & $B$ & $\sigma_{B}$ & $V$ & $\sigma_{V}$ & $R$ & $\sigma_{R}$ & $I$ & $\sigma_{I}$ \\
\hline $\mathrm{c} 6$ & 16.878 & 0.003 & 15.941 & 0.005 & 15.409 & 0.002 & 14.925 & $0.00^{\circ}$ \\
\hline c7 & 18.070 & 0.015 & 17.546 & $\begin{array}{c}0.008 \\
\text { SN 1992J }\end{array}$ & 17.192 & 0.007 & 16.843 & 0.01 \\
\hline $\mathrm{cl}$ & 15.341 & 0.005 & 14.449 & 0.001 & $\ldots$ & $\cdots$ & 13.385 & 0.00 \\
\hline $\mathrm{c} 2$ & 19.538 & 0.005 & 18.149 & 0.003 & $\ldots$ & $\cdots$ & 16.520 & $0.00^{\circ}$ \\
\hline c3 & 18.258 & 0.008 & 17.305 & 0.001 & $\ldots$ & $\ldots$ & 16.164 & 0.00 \\
\hline $\mathrm{c} 4$ & 17.840 & 0.002 & 17.168 & 0.003 & $\ldots$ & $\ldots$ & 16.400 & $0.00 t$ \\
\hline c5 & 21.701 & 0.018 & 20.027 & 0.003 & $\ldots$ & $\cdots$ & 17.544 & $0.01 \mathrm{t}$ \\
\hline c6 & 18.773 & 0.004 & 18.110 & 0.001 & $\ldots$ & $\ldots$ & 17.264 & 0.011 \\
\hline c7 & 20.381 & 0.009 & 18.905 & 0.004 & $\ldots$ & $\ldots$ & 17.060 & 0.01 : \\
\hline $\mathrm{c} 8$ & 18.671 & 0.002 & 17.956 & $\begin{array}{c}0.006 \\
\text { SN 1992K }\end{array}$ & $\ldots$ & $\ldots$ & 17.127 & $0.01:$ \\
\hline $\mathrm{c} 1$ & 16.437 & 0.018 & 15.753 & 0.010 & $\ldots$ & $\ldots$ & 14.944 & 0.01 s \\
\hline $\mathrm{c} 2$ & 17.639 & 0.008 & 16.990 & 0.007 & $\ldots$ & $\ldots$ & 16.229 & $0.00^{*}$ \\
\hline c3 & 18.122 & 0.010 & 17.467 & 0.004 & $\ldots$ & $\ldots$ & 16.704 & 0.01( \\
\hline $\mathrm{c} 4$ & 17.798 & 0.010 & 17.134 & 0.002 & $\ldots$ & $\ldots$ & 16.368 & 0.00 \\
\hline $\mathrm{c} 5$ & 17.643 & 0.012 & 16.667 & 0.003 & $\ldots$ & $\ldots$ & 15.598 & $0.00^{\circ}$ \\
\hline$c 6$ & 17.139 & 0.010 & 16.490 & $\begin{array}{c}0.002 \\
\text { SN 1992P }\end{array}$ & $\ldots$ & $\ldots$ & 15.738 & $0.01:$ \\
\hline $\mathrm{c} 1$ & 16.985 & 0.004 & 16.335 & 0.008 & $\cdots$ & $\cdots$ & 15.631 & $0.00\}$ \\
\hline $\mathrm{c} 2$ & 17.997 & 0.011 & 16.512 & 0.007 & $\ldots$ & $\ldots$ & 14.510 & 0.011 \\
\hline $\mathrm{c} 3$ & 16.881 & 0.028 & 16.117 & 0.016 & $\ldots$ & $\ldots$ & 15.266 & 0.014 \\
\hline $\mathrm{c} 4$ & 17.809 & 0.017 & 17.047 & 0.015 & $\ldots$ & $\ldots$ & 16.177 & $0.01:$ \\
\hline$c 5$ & 19.646 & 0.007 & 18.541 & 0.014 & $\ldots$ & $\ldots$ & 17.202 & $0.00 \leq$ \\
\hline $\mathrm{c} 6$ & 19.024 & 0.019 & 17.766 & 0.018 & $\ldots$ & $\ldots$ & 16.375 & $0.01 \%$ \\
\hline c7 & 18.711 & 0.012 & 18.265 & 0.013 & $\ldots$ & $\ldots$ & 17.647 & $0.01:$ \\
\hline $\mathrm{c} 8$ & 19.573 & 0.027 & 18.050 & 0.012 & $\ldots$ & $\ldots$ & 15.506 & 0.00 s \\
\hline $\mathrm{c} 10$ & 20.313 & 0.038 & 19.321 & $\begin{array}{c}0.022 \\
\text { SN 1992ae }\end{array}$ & $\ldots$ & $\ldots$ & 18.154 & 0.01 \\
\hline $\mathrm{c} 1$ & 17.086 & 0.005 & 16.451 & 0.002 & $\ldots$ & $\ldots$ & 15.707 & 0.00 \\
\hline $\mathrm{c} 2$ & 15.720 & 0.009 & 14.782 & 0.006 & $\ldots$ & $\ldots$ & 13.789 & $0.00^{t}$ \\
\hline $\mathrm{c} 3$ & 19.772 & 0.025 & 18.585 & 0.007 & $\ldots$ & $\ldots$ & 17.231 & 0.00\{ \\
\hline $\mathrm{c} 4$ & 19.097 & 0.014 & 18.381 & 0.008 & $\ldots$ & $\ldots$ & 17.557 & $0.01 c$ \\
\hline$c 5$ & 19.464 & 0.120 & 18.977 & 0.058 & $\ldots$ & $\ldots$ & 18.200 & 0.10 \\
\hline $\mathrm{c} 6$ & 17.347 & 0.006 & 16.617 & 0.002 & $\cdots$ & $\ldots$ & 15.769 & 0.00 \\
\hline c7 & 16.719 & 0.007 & 16.300 & 0.004 & $\ldots$ & $\ldots$ & 15.774 & $0.00=$ \\
\hline $\mathrm{c} 8$ & 18.469 & 0.009 & 17.722 & $\begin{array}{c}0.009 \\
\text { SN 1992ag }\end{array}$ & $\ldots$ & $\ldots$ & 16.873 & $0.00 t$ \\
\hline $\mathrm{c} 1$ & 17.657 & 0.009 & 17.002 & 0.011 & $\ldots$ & $\ldots$ & 16.217 & 0.01 ; \\
\hline $\mathrm{c} 2$ & 18.203 & 0.009 & 17.465 & 0.010 & $\ldots$ & $\ldots$ & 16.594 & $0.02<$ \\
\hline c3 & 19.434 & 0.007 & 18.445 & 0.017 & $\ldots$ & $\ldots$ & 17.271 & $0.01 c$ \\
\hline $\mathrm{c} 4$ & 17.218 & 0.014 & 16.490 & 0.006 & $\cdots$ & $\ldots$ & 15.647 & 0.03 \\
\hline $\mathrm{c} 5$ & 18.731 & 0.035 & 18.111 & 0.022 & $\ldots$ & $\ldots$ & 17.318 & $0.00 t$ \\
\hline c6 & 17.726 & 0.007 & 16.819 & 0.006 & $\ldots$ & $\ldots$ & 15.750 & 0.011 \\
\hline c7 & 17.539 & 0.006 & 16.826 & 0.007 & $\ldots$ & $\ldots$ & 16.021 & $0.01 \varepsilon$ \\
\hline $\mathrm{c} 9$ & 18.445 & 0.016 & 17.938 & 0.010 & $\ldots$ & $\ldots$ & 17.221 & $0.01 \epsilon$ \\
\hline $\mathrm{c} 10$ & 17.802 & 0.008 & 17.159 & 0.010 & $\ldots$ & $\ldots$ & 16.378 & 0.015 \\
\hline $\mathrm{cll}$ & 18.607 & 0.011 & 17.921 & 0.013 & $\ldots$ & $\ldots$ & 17.081 & $0.01<$ \\
\hline c12 & 17.886 & 0.006 & 16.362 & $\begin{array}{l}0.011 \\
\text { SN 1992al }\end{array}$ & $\ldots$ & $\cdots$ & 14.407 & $0.01]$ \\
\hline $\mathrm{c} 1$ & 16.671 & 0.003 & 15.781 & 0.004 & 15.274 & 0.003 & 14.859 & $0.00 t$ \\
\hline $\mathrm{c} 2$ & 18.290 & 0.002 & 17.650 & 0.001 & 17.270 & 0.001 & 16.916 & $0.00^{c}$ \\
\hline $\mathrm{c} 3$ & 16.028 & 0.006 & 15.206 & 0.004 & 14.735 & 0.002 & 14.279 & $0.00^{c}$ \\
\hline $\mathrm{c} 4$ & 16.096 & 0.003 & 15.490 & 0.003 & 15.124 & 0.001 & 14.780 & $0.00=$ \\
\hline $\mathrm{c} 5$ & 16.674 & 0.003 & 16.067 & 0.002 & 15.713 & 0.002 & 15.353 & $0.00<$ \\
\hline c6 & 19.006 & 0.001 & 17.570 & 0.001 & 16.652 & 0.002 & 15.744 & 0.00 \\
\hline $\mathrm{c} 7$ & 16.937 & 0.005 & 16.080 & 0.004 & 15.555 & 0.003 & 15.078 & 0.00 \\
\hline $\mathrm{c} 8$ & 18.464 & 0.002 & 17.683 & 0.001 & 17.204 & 0.001 & 16.751 & $0.00^{\prime}$ \\
\hline c9 & 18.153 & 0.002 & 17.248 & $\begin{array}{l}0.001 \\
\text { SN 1992aq }\end{array}$ & 16.705 & 0.001 & 16.224 & 0.001 \\
\hline $\mathrm{c} 1$ & 15.748 & 0.003 & 15.138 & 0.004 & $\ldots$ & $\ldots$ & 14.422 & $0.00 \leqslant$ \\
\hline $\mathrm{c} 2$ & 15.400 & 0.007 & 14.502 & 0.004 & $\ldots$ & $\ldots$ & 13.530 & $0.00 \leqslant$ \\
\hline c3 & 20.677 & 0.019 & 19.114 & 0.006 & $\ldots$ & $\ldots$ & 17.092 & $0.00=$ \\
\hline $\mathrm{c} 4$ & 20.126 & 0.037 & 19.233 & 0.021 & $\ldots$ & $\ldots$ & 18.124 & 0.02( \\
\hline c5 & 18.994 & 0.013 & 17.626 & 0.004 & $\ldots$ & $\ldots$ & 15.985 & $0.00^{\prime}$ \\
\hline c7 & 21.066 & 0.070 & 19.650 & 0.002 & $\ldots$ & $\ldots$ & 17.923 & 0.03 \\
\hline$c 8$ & 16.590 & 0.010 & 15.146 & 0.010 & $\ldots$ & $\ldots$ & 13.735 & $0.00<$ \\
\hline c9 & 19.715 & 0.011 & 19.194 & 0.008 & $\ldots$ & $\ldots$ & 18.608 & 0.01 s \\
\hline
\end{tabular}


TABLE 3. (continued)

\begin{tabular}{|c|c|c|c|c|c|c|c|c|}
\hline Star & $B$ & $\sigma_{B}$ & $V$ & $\sigma_{V}$ & $R$ & $\sigma_{R}$ & $I$ & $\sigma_{I}$ \\
\hline $\mathrm{c} 10$ & 15.275 & 0.006 & 14.481 & 0.010 & $\cdots$ & $\cdots$ & 13.595 & 0.010 \\
\hline $\mathrm{c} 11$ & 17.277 & 0.010 & 16.645 & $\begin{array}{c}0.010 \\
\text { SN 1992au }\end{array}$ & $\cdots$ & $\cdots$ & 15.910 & 0.003 \\
\hline $\mathrm{c} 2$ & 17.421 & 0.020 & 16.030 & 0.006 & $\cdots$ & $\ldots$ & 14.117 & 0.005 \\
\hline $\mathrm{c} 3$ & 16.056 & 0.006 & 15.386 & 0.007 & $\cdots$ & $\ldots$ & 14.605 & 0.007 \\
\hline $\mathrm{c} 4$ & 19.131 & 0.013 & 17.698 & $\begin{array}{c}0.004 \\
\text { SN } 1992 b c\end{array}$ & $\cdots$ & $\cdots$ & 15.666 & 0.003 \\
\hline $\mathrm{c} 1$ & 14.213 & 0.008 & 13.570 & 0.006 & $\ldots$ & $\ldots$ & 12.862 & 0.011 \\
\hline $\mathrm{c} 2$ & 14.297 & 0.005 & 13.645 & 0.004 & 13.280 & 0.001 & 12.909 & 0.008 \\
\hline c3 & 15.785 & 0.003 & 15.060 & 0.003 & 14.644 & 0.003 & 14.255 & 0.003 \\
\hline $\mathrm{c} 4$ & 17.756 & 0.005 & 17.288 & 0.005 & 16.986 & 0.003 & 16.682 & 0.007 \\
\hline$c 5$ & 16.673 & 0.004 & 15.659 & 0.005 & 15.029 & 0.004 & 14.487 & 0.005 \\
\hline c6 & 17.087 & 0.026 & 16.422 & 0.012 & 16.028 & 0.010 & 15.647 & 0.004 \\
\hline c7 & 17.244 & 0.006 & 16.523 & 0.013 & 16.094 & 0.014 & 15.688 & 0.016 \\
\hline$c 8$ & 20.514 & 0.089 & 19.052 & 0.025 & 17.990 & 0.031 & 16.660 & 0.024 \\
\hline c9 & 19.858 & 0.057 & 18.430 & 0.001 & 17.530 & 0.001 & 16.678 & 0.002 \\
\hline $\mathrm{c} 10$ & 21.214 & 0.083 & 19.878 & $\begin{array}{c}0.039 \\
\text { SN } 1992 b g\end{array}$ & 19.156 & 0.005 & 18.491 & 0.076 \\
\hline $\mathrm{c} 1$ & 16.454 & 0.006 & 15.451 & 0.002 & $\cdots$ & $\ldots$ & 14.415 & 0.005 \\
\hline $\mathrm{c} 2$ & 16.830 & 0.006 & 16.173 & 0.005 & $\ldots$ & $\ldots$ & 15.399 & 0.003 \\
\hline c3 & 16.105 & 0.005 & 15.420 & 0.003 & $\cdots$ & $\cdots$ & 14.613 & 0.003 \\
\hline c6 & 18.897 & 0.020 & 17.797 & 0.011 & $\ldots$ & $\ldots$ & 16.566 & 0.003 \\
\hline c7 & 18.353 & 0.009 & 17.490 & 0.003 & $\ldots$ & $\ldots$ & 16.504 & 0.004 \\
\hline $\mathrm{c} 8$ & 19.386 & 0.016 & 18.362 & 0.003 & $\ldots$ & $\cdots$ & 17.288 & 0.005 \\
\hline c9 & 20.428 & 0.047 & 19.680 & 0.011 & $\cdots$ & $\cdots$ & 18.485 & 0.023 \\
\hline $\mathrm{c} 10$ & 20.345 & 0.038 & 19.094 & $\begin{array}{c}0.007 \\
\text { SN 1992bh }\end{array}$ & $\ldots$ & $\ldots$ & 17.708 & 0.011 \\
\hline $\mathrm{c} 1$ & 17.920 & 0.008 & 17.296 & 0.007 & $\ldots$ & $\ldots$ & 16.551 & 0.010 \\
\hline c2 & 19.202 & 0.012 & 17.913 & 0.006 & $\ldots$ & $\ldots$ & 16.411 & 0.009 \\
\hline c3 & 17.265 & 0.003 & 16.403 & 0.002 & $\cdots$ & $\cdots$ & 15.433 & 0.005 \\
\hline $\mathrm{c} 4$ & 18.937 & 0.007 & 18.618 & 0.006 & $\cdots$ & $\cdots$ & 18.178 & 0.021 \\
\hline $\mathrm{c} 5$ & 18.095 & 0.004 & 17.484 & 0.003 & $\ldots$ & $\ldots$ & 16.760 & 0.003 \\
\hline c6 & 18.543 & 0.014 & 17.807 & 0.003 & $\cdots$ & $\cdots$ & 16.930 & 0.006 \\
\hline c7 & 20.749 & 0.025 & 19.994 & 0.020 & $\ldots$ & $\ldots$ & 19.031 & 0.026 \\
\hline c8 & 20.621 & 0.021 & 19.324 & 0.014 & $\cdots$ & $\cdots$ & 17.766 & 0.008 \\
\hline c9 & 16.390 & 0.010 & 15.461 & $\begin{array}{c}0.010 \\
\text { SN } 1992 \mathrm{bk}\end{array}$ & $\ldots$ & $\cdots$ & $\cdots$ & $\cdots$ \\
\hline $\mathrm{c} 1$ & 18.003 & 0.006 & 17.410 & 0.008 & $\ldots$ & $\ldots$ & 16.685 & 0.004 \\
\hline c3 & 17.851 & 0.007 & 16.809 & 0.005 & $\cdots$ & $\cdots$ & 15.577 & 0.004 \\
\hline$c 4$ & 15.430 & 0.007 & 14.708 & 0.016 & $\cdots$ & $\ldots$ & 13.894 & 0.010 \\
\hline c6 & 20.578 & 0.018 & 19.002 & 0.006 & $\ldots$ & $\ldots$ & 16.950 & 0.005 \\
\hline $\mathrm{c} 12$ & 18.764 & 0.010 & 17.669 & $\begin{array}{c}0.009 \\
\text { SN } 1992 \mathrm{bl}\end{array}$ & $\cdots$ & $\ldots$ & 16.381 & 0.010 \\
\hline $\mathrm{cl}$ & 15.285 & 0.010 & 14.500 & 0.010 & $\cdots$ & $\ldots$ & 13.540 & 0.010 \\
\hline $\mathrm{c} 2$ & 16.400 & 0.003 & 15.608 & 0.005 & $\ldots$ & $\ldots$ & 14.766 & 0.002 \\
\hline $\mathrm{c} 4$ & 15.136 & 0.007 & 14.564 & 0.005 & $\ldots$ & $\cdots$ & 13.882 & 0.002 \\
\hline$c 5$ & 19.746 & 0.002 & 19.187 & 0.003 & $\cdots$ & $\ldots$ & 18.504 & 0.009 \\
\hline c7 & 16.939 & 0.003 & 16.419 & 0.002 & $\ldots$ & $\ldots$ & 15.759 & 0.003 \\
\hline$c 8$ & 18.820 & 0.008 & 18.220 & $\begin{array}{c}0.003 \\
\text { SN 1992bo }\end{array}$ & $\ldots$ & $\ldots$ & 17.506 & 0.002 \\
\hline $\mathrm{c} 2$ & 18.063 & 0.018 & 16.930 & 0.005 & 16.231 & 0.007 & 15.687 & 0.005 \\
\hline c3 & 19.882 & 0.025 & 19.365 & 0.006 & 18.992 & 0.012 & 18.634 & 0.016 \\
\hline $\mathrm{c} 4$ & 20.702 & 0.050 & 19.199 & 0.013 & 18.262 & 0.005 & 17.291 & 0.011 \\
\hline$c 5$ & 15.348 & 0.011 & 14.470 & 0.007 & 13.973 & 0.006 & 13.545 & 0.007 \\
\hline c7 & 16.916 & 0.010 & 16.373 & 0.003 & 16.007 & 0.005 & 15.713 & 0.005 \\
\hline$c 8$ & 20.752 & 0.059 & 19.930 & $\begin{array}{c}0.016 \\
\text { SN } 1992 b p\end{array}$ & 19.530 & 0.017 & 18.949 & 0.026 \\
\hline$c 2$ & 20.291 & 0.012 & 18.737 & 0.005 & $\ldots$ & $\ldots$ & 16.455 & 0.005 \\
\hline c3 & 14.344 & 0.015 & 13.691 & 0.012 & $\cdots$ & $\ldots$ & $\cdots$ & $\cdots$ \\
\hline $\mathrm{c} 4$ & 21.587 & 0.024 & 19.974 & 0.010 & $\cdots$ & $\ldots$ & 17.327 & 0.050 \\
\hline c5 & 21.756 & 0.032 & 20.540 & 0.016 & $\ldots$ & $\ldots$ & 18.848 & 0.023 \\
\hline c6 & 17.718 & 0.005 & 17.080 & 0.003 & $\cdots$ & $\ldots$ & 16.312 & 0.006 \\
\hline c9 & 21.014 & 0.017 & 20.512 & $\begin{array}{c}0.017 \\
\text { SN 1992br }\end{array}$ & $\ldots$ & $\ldots$ & 20.010 & 0.028 \\
\hline $\mathrm{cl}$ & 18.042 & 0.004 & 17.316 & 0.004 & $\ldots$ & $\ldots$ & $\ldots$ & $\cdots$ \\
\hline $\mathrm{c} 2$ & 17.555 & 0.002 & 16.870 & 0.002 & $\cdots$ & $\ldots$ & $\cdots$ & $\cdots$ \\
\hline c3 & 19.428 & 0.005 & 18.728 & 0.007 & $\cdots$ & $\cdots$ & $\cdots$ & $\cdots$ \\
\hline c4 & 20.373 & 0.007 & 19.759 & 0.010 & $\ldots$ & $\ldots$ & $\ldots$ & $\ldots$ \\
\hline
\end{tabular}


TABLE 3. (continued)

\begin{tabular}{|c|c|c|c|c|c|c|c|c|}
\hline Star & $B$ & $\sigma_{B}$ & $V$ & $\sigma_{V}$ & $R$ & $\sigma_{R}$ & $I$ & $\sigma_{I}$ \\
\hline \multicolumn{9}{|c|}{ SN 1992bs } \\
\hline $\mathrm{c} 1$ & 16.801 & 0.005 & 16.234 & 0.004 & $\ldots$ & $\ldots$ & $\ldots$ & $\ldots$ \\
\hline $\mathrm{c} 2$ & 19.433 & 0.014 & 18.520 & 0.006 & $\cdots$ & $\cdots$ & $\cdots$ & $\cdots$ \\
\hline c3 & 17.038 & 0.005 & 16.211 & 0.004 & $\ldots$ & $\ldots$ & $\cdots$ & $\ldots$ \\
\hline $\mathrm{c} 4$ & 16.447 & 0.005 & 15.800 & 0.004 & $\ldots$ & $\ldots$ & $\ldots$ & $\ldots$ \\
\hline$c 5$ & 17.589 & 0.007 & 16.708 & 0.004 & $\ldots$ & $\ldots$ & $\ldots$ & $\cdots$ \\
\hline c6 & 14.955 & 0.007 & 14.278 & 0.011 & $\ldots$ & $\ldots$ & $\ldots$ & $\ldots$ \\
\hline c7 & 20.912 & 0.033 & 19.580 & 0.019 & $\cdots$ & $\cdots$ & $\ldots$ & $\cdots$ \\
\hline $\mathrm{c} 8$ & 21.127 & 0.040 & 19.601 & 0.017 & $\cdots$ & $\cdots$ & $\cdots$ & $\cdots$ \\
\hline \multicolumn{9}{|c|}{ SN 1993B } \\
\hline $\mathrm{c} 1$ & 18.762 & 0.006 & 18.149 & 0.004 & $\ldots$ & $\ldots$ & 17.419 & 0.006 \\
\hline$c 2$ & 16.590 & 0.005 & 15.650 & 0.004 & $\ldots$ & $\cdots$ & 14.617 & 0.004 \\
\hline c3 & 18.333 & 0.005 & 17.309 & 0.004 & $\ldots$ & $\ldots$ & 16.243 & 0.002 \\
\hline $\mathrm{c} 4$ & 19.222 & 0.006 & 18.090 & 0.003 & $\ldots$ & $\ldots$ & 16.853 & 0.003 \\
\hline $\mathrm{c} 5$ & 19.195 & 0.005 & 17.805 & 0.004 & $\ldots$ & $\ldots$ & 16.171 & 0.004 \\
\hline c6 & 16.116 & 0.004 & 15.657 & 0.003 & $\cdots$ & $\cdots$ & 15.011 & 0.004 \\
\hline c7 & 17.630 & 0.005 & 16.817 & 0.003 & $\ldots$ & $\ldots$ & 15.949 & 0.003 \\
\hline $\mathrm{c} 8$ & 15.968 & 0.003 & 15.289 & 0.003 & $\ldots$ & $\ldots$ & 14.523 & 0.003 \\
\hline$c 9$ & 15.630 & 0.004 & 14.982 & 0.003 & $\ldots$ & $\ldots$ & 14.232 & 0.004 \\
\hline $\mathrm{c} 10$ & 18.536 & 0.007 & 17.629 & 0.005 & $\ldots$ & $\ldots$ & 16.591 & 0.006 \\
\hline \multicolumn{9}{|c|}{ SN $1993 \mathrm{H}$} \\
\hline $\mathrm{cl}$ & 15.891 & 0.012 & 15.265 & 0.005 & 14.928 & 0.020 & 14.552 & 0.005 \\
\hline $\mathrm{c} 2$ & 15.874 & 0.014 & 14.602 & 0.011 & $\cdots$ & $\cdots$ & 13.193 & 0.015 \\
\hline c3 & 15.468 & 0.016 & 14.745 & 0.013 & 14.372 & 0.010 & 13.977 & 0.008 \\
\hline $\mathrm{c} 4$ & 18.802 & 0.006 & 18.089 & 0.004 & 17.666 & 0.005 & 17.264 & 0.005 \\
\hline$c 5$ & 19.148 & 0.003 & 17.719 & 0.005 & 16.834 & 0.006 & 15.968 & 0.005 \\
\hline c6 & 18.506 & 0.005 & 17.037 & 0.002 & 16.066 & 0.004 & 15.007 & 0.005 \\
\hline c7 & 17.277 & 0.007 & 16.334 & 0.004 & 15.831 & 0.011 & 15.358 & 0.006 \\
\hline $\mathrm{c} 8$ & 16.742 & 0.014 & 15.546 & 0.010 & 14.833 & 0.013 & 14.180 & 0.003 \\
\hline c9 & 17.223 & 0.007 & 16.677 & 0.003 & 16.326 & 0.005 & 15.979 & 0.003 \\
\hline $\mathrm{c} 10$ & 18.724 & 0.010 & 18.029 & 0.012 & 17.612 & 0.013 & 17.201 & 0.004 \\
\hline c11 & 19.952 & 0.010 & 19.082 & 0.015 & 18.535 & 0.010 & 18.032 & 0.008 \\
\hline $\mathrm{c} 12$ & 19.279 & 0.003 & 18.475 & 0.005 & 17.996 & 0.009 & 17.554 & 0.006 \\
\hline \multicolumn{9}{|c|}{ SN 19930} \\
\hline $\mathrm{c} 1$ & 18.648 & 0.024 & 17.362 & 0.024 & $\cdots$ & $\cdots$ & 15.757 & 0.021 \\
\hline $\mathrm{c} 2$ & 18.728 & 0.015 & 18.045 & 0.023 & $\ldots$ & $\ldots$ & 17.211 & 0.021 \\
\hline c3 & 18.752 & 0.018 & 18.028 & 0.023 & $\ldots$ & $\cdots$ & 17.194 & 0.029 \\
\hline $\mathrm{c} 4$ & 19.141 & 0.023 & 18.143 & 0.018 & $\cdots$ & $\ldots$ & 17.046 & 0.023 \\
\hline $\mathrm{c} 5$ & 19.911 & 0.020 & 19.236 & 0.015 & $\ldots$ & $\ldots$ & 18.493 & 0.010 \\
\hline$c 6$ & 19.719 & 0.022 & 18.671 & 0.029 & $\cdots$ & $\cdots$ & 17.430 & 0.010 \\
\hline c7 & 15.638 & 0.009 & 15.000 & 0.027 & $\cdots$ & $\cdots$ & 14.222 & 0.013 \\
\hline $\mathrm{c} 8$ & 16.260 & 0.013 & 15.306 & 0.038 & $\cdots$ & $\cdots$ & 14.187 & 0.014 \\
\hline c9 & 17.885 & 0.017 & 17.014 & 0.018 & $\cdots$ & $\cdots$ & 16.080 & 0.020 \\
\hline $\mathrm{c} 10$ & 18.105 & 0.006 & 17.327 & 0.004 & $\cdots$ & $\cdots$ & 16.441 & 0.016 \\
\hline $\mathrm{c} 11$ & 18.386 & 0.022 & 17.718 & 0.044 & $\ldots$ & $\cdots$ & 16.908 & 0.028 \\
\hline $\mathrm{c} 12$ & 18.468 & 0.026 & 17.814 & 0.026 & $\cdots$ & $\cdots$ & 16.960 & 0.026 \\
\hline \multicolumn{9}{|c|}{ SN 1993ag } \\
\hline $\mathrm{cl}$ & 15.680 & 0.004 & 15.041 & 0.003 & $\cdots$ & $\cdots$ & 14.329 & 0.003 \\
\hline$c 2$ & 15.415 & 0.004 & 14.672 & 0.003 & $\cdots$ & $\cdots$ & 13.878 & 0.003 \\
\hline c3 & 18.349 & 0.005 & 17.432 & 0.003 & $\ldots$ & $\ldots$ & 16.466 & 0.006 \\
\hline $\mathrm{c} 4$ & 18.710 & 0.007 & 17.880 & 0.004 & $\ldots$ & $\ldots$ & 16.988 & 0.006 \\
\hline$c 5$ & 19.536 & 0.018 & 18.745 & 0.007 & $\cdots$ & $\cdots$ & 17.877 & 0.019 \\
\hline c6 & 20.138 & 0.022 & 18.626 & 0.005 & $\ldots$ & $\ldots$ & 16.311 & 0.005 \\
\hline c7 & 20.808 & 0.023 & 20.218 & 0.009 & $\cdots$ & $\cdots$ & 19.514 & 0.027 \\
\hline $\mathrm{c} 8$ & 20.643 & 0.024 & 19.181 & 0.007 & $\cdots$ & $\cdots$ & 16.975 & 0.006 \\
\hline $\mathrm{c} 9$ & 19.733 & 0.005 & 18.952 & 0.005 & $\ldots$ & $\ldots$ & 18.095 & 0.008 \\
\hline $\mathrm{c} 10$ & 18.728 & 0.007 & 17.843 & 0.005 & $\cdots$ & $\cdots$ & 16.855 & 0.006 \\
\hline \multicolumn{9}{|c|}{ SN 1993ah } \\
\hline $\mathrm{c} 1$ & 17.875 & 0.006 & 16.861 & 0.002 & $\cdots$ & $\cdots$ & 15.726 & 0.005 \\
\hline$c 2$ & 19.672 & 0.005 & 18.164 & 0.003 & $\cdots$ & $\cdots$ & 16.122 & 0.001 \\
\hline c3 & 17.265 & 0.016 & 16.763 & 0.015 & $\cdots$ & $\cdots$ & 16.105 & 0.016 \\
\hline $\mathrm{c} 4$ & 18.538 & 0.008 & 17.089 & 0.001 & $\cdots$ & $\cdots$ & 15.085 & 0.001 \\
\hline$c 5$ & 18.488 & 0.004 & 17.482 & 0.002 & $\cdots$ & $\cdots$ & 16.384 & 0.003 \\
\hline$c 6$ & 19.518 & 0.002 & 18.586 & 0.003 & $\ldots$ & $\cdots$ & 17.503 & 0.004 \\
\hline c7 & 19.923 & 0.004 & 18.919 & 0.003 & $\cdots$ & $\cdots$ & 17.724 & 0.004 \\
\hline $\mathrm{c} 8$ & 18.369 & 0.005 & 17.657 & 0.001 & $\cdots$ & $\cdots$ & 16.859 & 0.002 \\
\hline
\end{tabular}


TABLE 4. $B V(R I)_{K C}$ photometry for the 29 SNe Ia.*

\begin{tabular}{|c|c|c|c|c|c|c|c|c|}
\hline $\begin{array}{c}\text { JD } \\
2440000+\end{array}$ & $B$ & $\sigma_{B}$ & $V$ & $\sigma_{V}$ & $R$ & $\sigma_{R}$ & $I$ & $\sigma_{I}$ \\
\hline \multicolumn{9}{|c|}{ SN 19900} \\
\hline 8076.71 & 16.600 & 0.030 & 16.532 & 0.030 & 16.413 & 0.030 & $\cdots$ & $\cdots$ \\
\hline 8084.64 & 16.956 & 0.030 & 16.699 & 0.030 & 16.646 & 0.030 & 17.126 & 0.030 \\
\hline 8095.67 & 18.019 & 0.036 & 17.278 & 0.033 & 17.196 & 0.031 & 17.435 & 0.034 \\
\hline 8104.67 & 18.928 & 0.048 & 17.759 & 0.036 & 17.326 & 0.031 & 17.243 & 0.035 \\
\hline 8104.67 & 18.929 & 0.053 & $\cdots$ & $\cdots$ & $\cdots$ & $\cdots$ & $\cdots$ & $\cdots$ \\
\hline 8115.64 & 19.549 & 0.048 & 18.376 & 0.034 & 17.918 & 0.033 & 17.735 & 0.037 \\
\hline 8126.48 & 19.760 & 0.068 & 18.681 & 0.041 & 18.302 & 0.036 & 18.216 & 0.055 \\
\hline 8149.58 & 20.104 & 0.067 & 19.279 & 0.041 & 18.955 & 0.044 & 19.556 & 0.151 \\
\hline 8163.52 & 20.402 & 0.233 & 19.400 & 0.100 & $\cdots$ & $\cdots$ & $\cdots$ & $\cdots$ \\
\hline 8353.83 & 22.830 & 0.598 & 22.529 & 0.312 & 22.952 & 0.514 & 21.613 & 0.497 \\
\hline 8355.86 & 22.747 & 0.155 & 22.965 & 0.160 & 23.155 & 0.184 & 22.471 & 0.215 \\
\hline 8355.86 & $\cdots$ & $\cdots$ & $\cdots$ & $\cdots$ & $\cdots$ & $\cdots$ & 23.238 & 0.359 \\
\hline 8383.88 & $\cdots$ & $\cdots$ & 22.886 & 0.316 & 23.629 & 0.785 & 22.368 & 0.817 \\
\hline \multicolumn{9}{|c|}{ SN 1990T } \\
\hline 8098.50 & 18.401 & 0.033 & 17.809 & 0.032 & 17.834 & 0.033 & 18.175 & 0.050 \\
\hline 8099.69 & 18.575 & 0.035 & 17.895 & 0.031 & 17.873 & 0.033 & 18.060 & 0.045 \\
\hline 8103.69 & $\cdots$ & $\cdots$ & 18.088 & 0.036 & $\cdots$ & $\cdots$ & 17.968 & 0.045 \\
\hline 8104.79 & 19.218 & 0.041 & 18.216 & 0.033 & 17.972 & 0.033 & 18.039 & 0.036 \\
\hline 8104.79 & 19.165 & 0.034 & 18.194 & 0.031 & 17.963 & 0.031 & 18.022 & 0.034 \\
\hline 8115.67 & 20.184 & 0.050 & 18.861 & 0.034 & 18.283 & 0.031 & 18.078 & 0.034 \\
\hline 8124.54 & 20.496 & 0.060 & 19.347 & 0.042 & 18.831 & 0.040 & 18.683 & 0.054 \\
\hline 8124.54 & $\cdots$ & $\cdots$ & $\cdots$ & $\cdots$ & 18.839 & 0.041 & $\cdots$ & $\cdots$ \\
\hline 8125.54 & 20.513 & 0.049 & 19.393 & 0.036 & 18.870 & 0.033 & 18.679 & 0.041 \\
\hline 8125.54 & 20.566 & 0.049 & 19.384 & 0.034 & $\cdots$ & $\cdots$ & $\cdots$ & $\cdots$ \\
\hline 8127.67 & 20.504 & 0.048 & 19.398 & 0.036 & 18.958 & 0.034 & 18.723 & 0.038 \\
\hline 8144.67 & 20.866 & 0.064 & 19.912 & 0.043 & 19.492 & 0.045 & 19.442 & 0.069 \\
\hline 8144.67 & $\cdots$ & $\cdots$ & $\cdots$ & $\cdots$ & 19.494 & 0.043 & 19.472 & 0.082 \\
\hline 8145.60 & 20.890 & 0.054 & 19.857 & 0.053 & 19.595 & 0.076 & 19.399 & 0.069 \\
\hline 8145.60 & $\cdots$ & $\cdots$ & $\cdots$ & $\cdots$ & 19.524 & 0.085 & $\cdots$ & $\cdots$ \\
\hline 8147.50 & 20.861 & 0.102 & 19.955 & 0.059 & 19.702 & 0.068 & 19.482 & 0.117 \\
\hline 8149.67 & 20.915 & 0.133 & 19.965 & 0.045 & 19.593 & 0.050 & $\cdots$ & $\cdots$ \\
\hline 8163.67 & 21.132 & 0.322 & 20.403 & 0.122 & 20.268 & 0.139 & $\cdots$ & $\cdots$ \\
\hline 8174.50 & 21.174 & 0.203 & 20.438 & 0.100 & 20.327 & 0.077 & 20.388 & 0.302 \\
\hline 8178.58 & 21.333 & 0.077 & 20.647 & 0.041 & 20.629 & 0.053 & 20.601 & 0.076 \\
\hline 8184.56 & 21.520 & 0.091 & 20.760 & 0.067 & 20.810 & 0.113 & $\cdots$ & $\cdots$ \\
\hline 8218.50 & 21.861 & 0.137 & 21.262 & 0.097 & $\cdots$ & $\cdots$ & $\cdots$ & $\cdots$ \\
\hline \multicolumn{9}{|c|}{ SN 1990Y } \\
\hline 8132.90 & 18.926 & 0.044 & 17.986 & 0.034 & 17.807 & 0.048 & $\cdots$ & $\cdots$ \\
\hline 8133.88 & 18.831 & 0.093 & $\cdots$ & $\cdots$ & $\cdots$ & $\cdots$ & $\cdots$ & $\cdots$ \\
\hline 8133.88 & 18.924 & 0.051 & 17.967 & 0.046 & $\cdots$ & $\cdots$ & $\cdots$ & $\cdots$ \\
\hline 8135.85 & 19.122 & 0.051 & 18.048 & 0.036 & $\cdots$ & $\cdots$ & $\cdots$ & $\cdots$ \\
\hline 8142.90 & 19.971 & 0.048 & 18.539 & 0.036 & $\cdots$ & $\cdots$ & $\cdots$ & $\cdots$ \\
\hline 8145.81 & 20.065 & 0.039 & 18.679 & 0.033 & 18.095 & 0.039 & 17.975 & 0.041 \\
\hline 8148.83 & 20.387 & 0.053 & 18.900 & 0.036 & 18.357 & 0.044 & 18.124 & 0.048 \\
\hline 8149.88 & 20.359 & 0.079 & 18.930 & 0.034 & 18.308 & 0.034 & 18.113 & 0.059 \\
\hline 8154.85 & 20.449 & 0.150 & 19.286 & 0.045 & 18.639 & 0.048 & 18.444 & 0.089 \\
\hline 8174.71 & $\cdots$ & $\cdots$ & 19.560 & 0.068 & 19.142 & 0.050 & 19.235 & 0.096 \\
\hline 8178.83 & 21.072 & 0.048 & $\cdots$ & $\cdots$ & 19.578 & 0.036 & 19.669 & 0.050 \\
\hline 8178.83 & 21.084 & 0.048 & 19.984 & 0.035 & 19.576 & 0.037 & 19.697 & 0.051 \\
\hline 8178.83 & 21.052 & 0.065 & 19.923 & 0.033 & 19.579 & 0.037 & 19.682 & 0.051 \\
\hline 8184.75 & 21.153 & 0.100 & 20.095 & 0.053 & $\cdots$ & $\cdots$ & $\cdots$ & $\cdots$ \\
\hline 8188.81 & 21.392 & 0.095 & 20.305 & 0.050 & 19.997 & 0.056 & 20.142 & 0.113 \\
\hline 8218.80 & 21.421 & 0.115 & 20.888 & 0.074 & $\cdots$ & $\cdots$ & $\cdots$ & $\cdots$ \\
\hline 8218.80 & 21.554 & 0.142 & $\cdots$ & $\cdots$ & $\cdots$ & $\cdots$ & $\cdots$ & $\cdots$ \\
\hline
\end{tabular}

*Table 4 can be found in the AAS CD-ROM Series. Only the first page is shown here for form and content. 

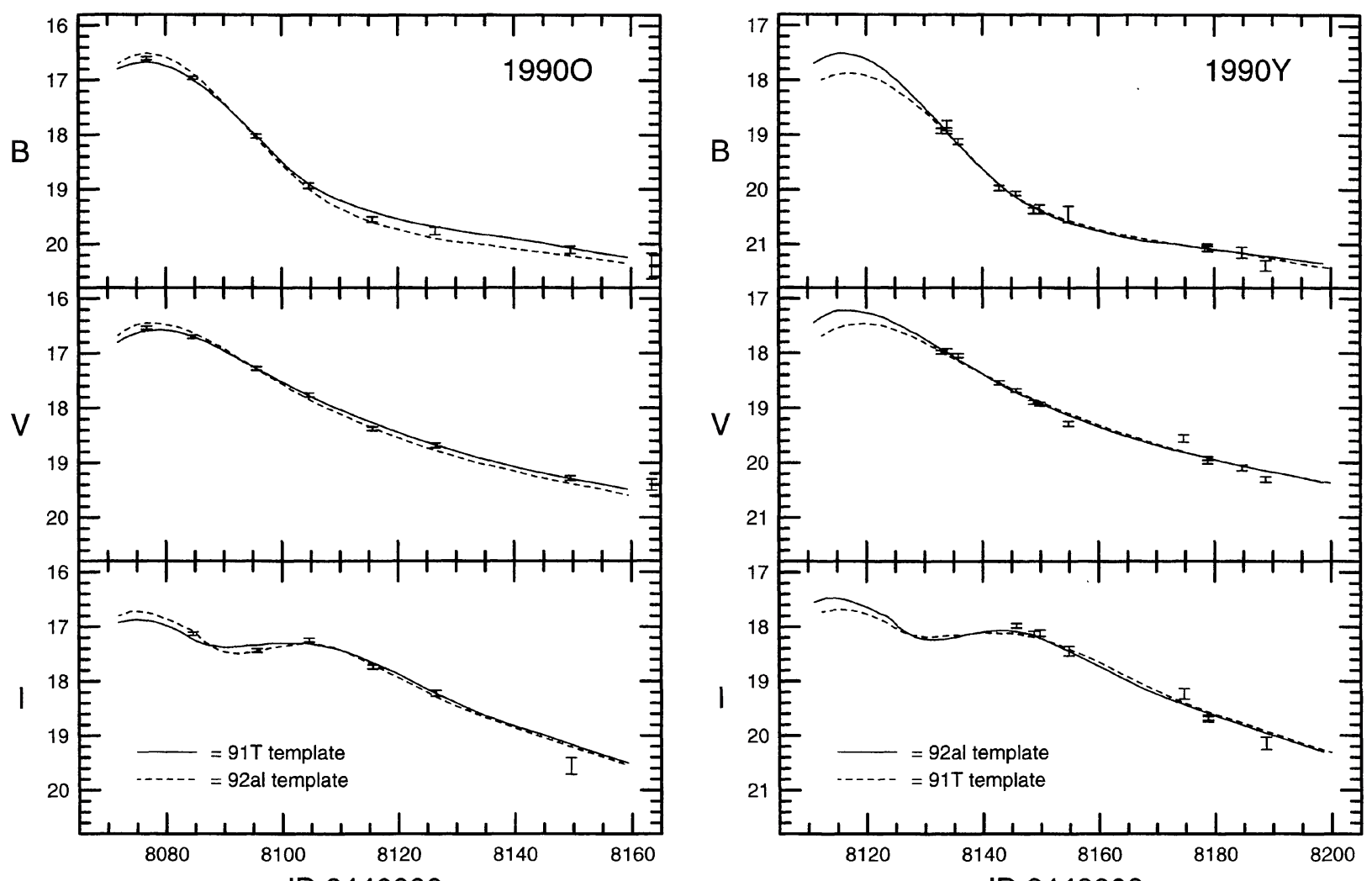

(a)

JD 2440000+

(c)

JD 2440000+
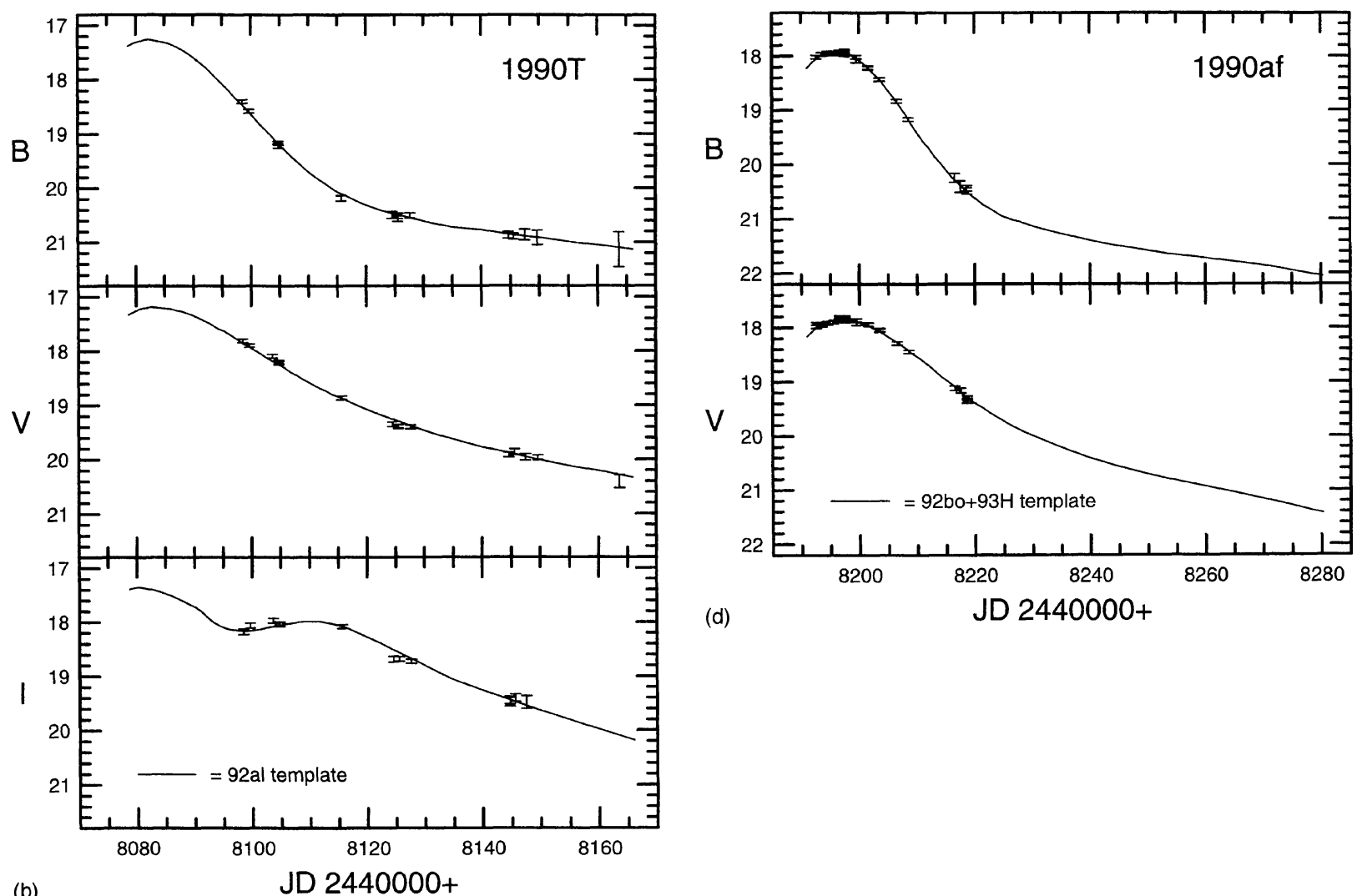

(d)

JD 2440000+

FIG. 3. $B, V$, and $I$ light curves for the $29 \mathrm{SNe}$ Ia. In all cases the solid lines correspond to the best-fitting template. The next-best fit is also shown as dashed lines when the difference in the reduced $\chi^{2}$ between the two best fits was $\leqslant 1.5$. 

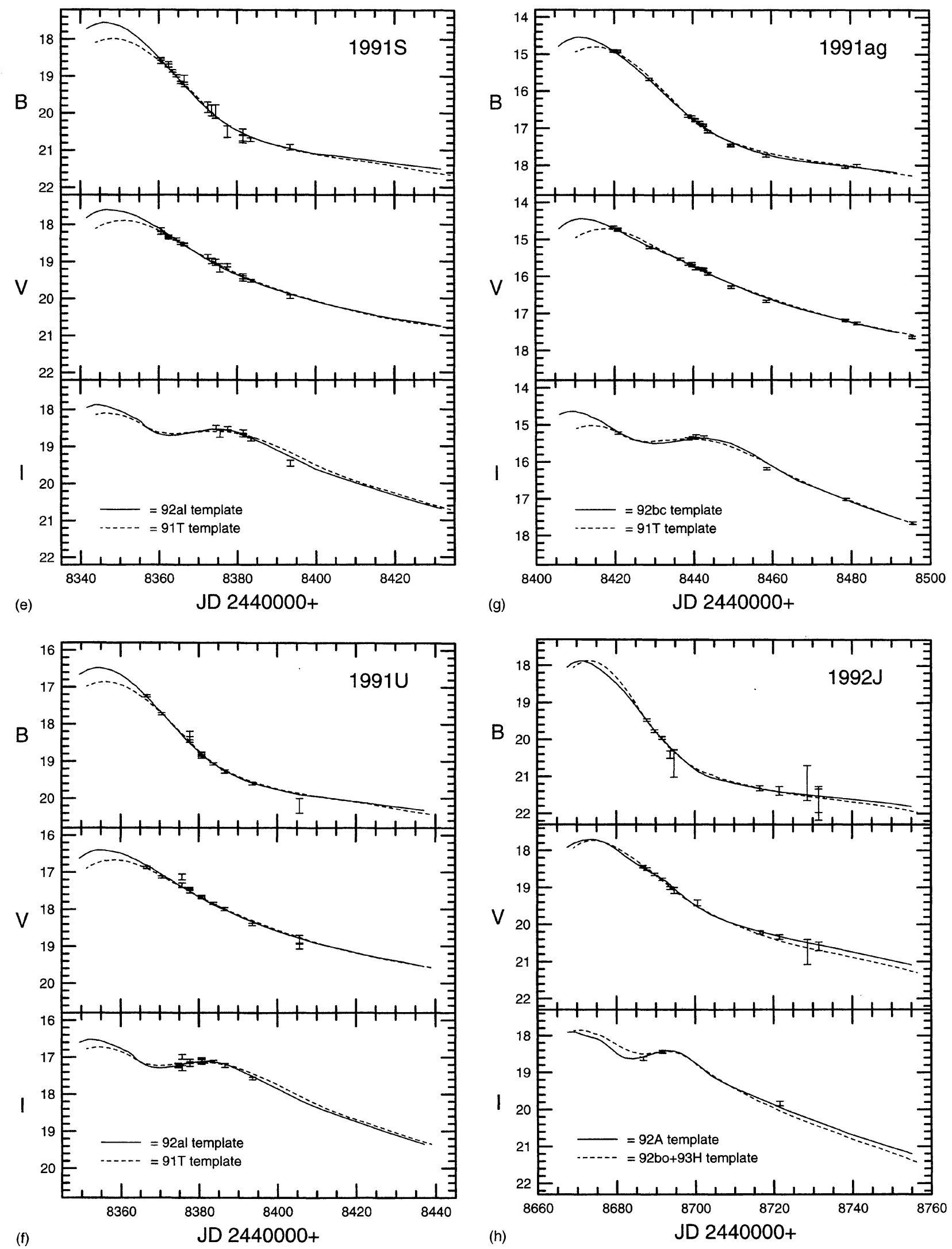

FIG. 3. (continued) 

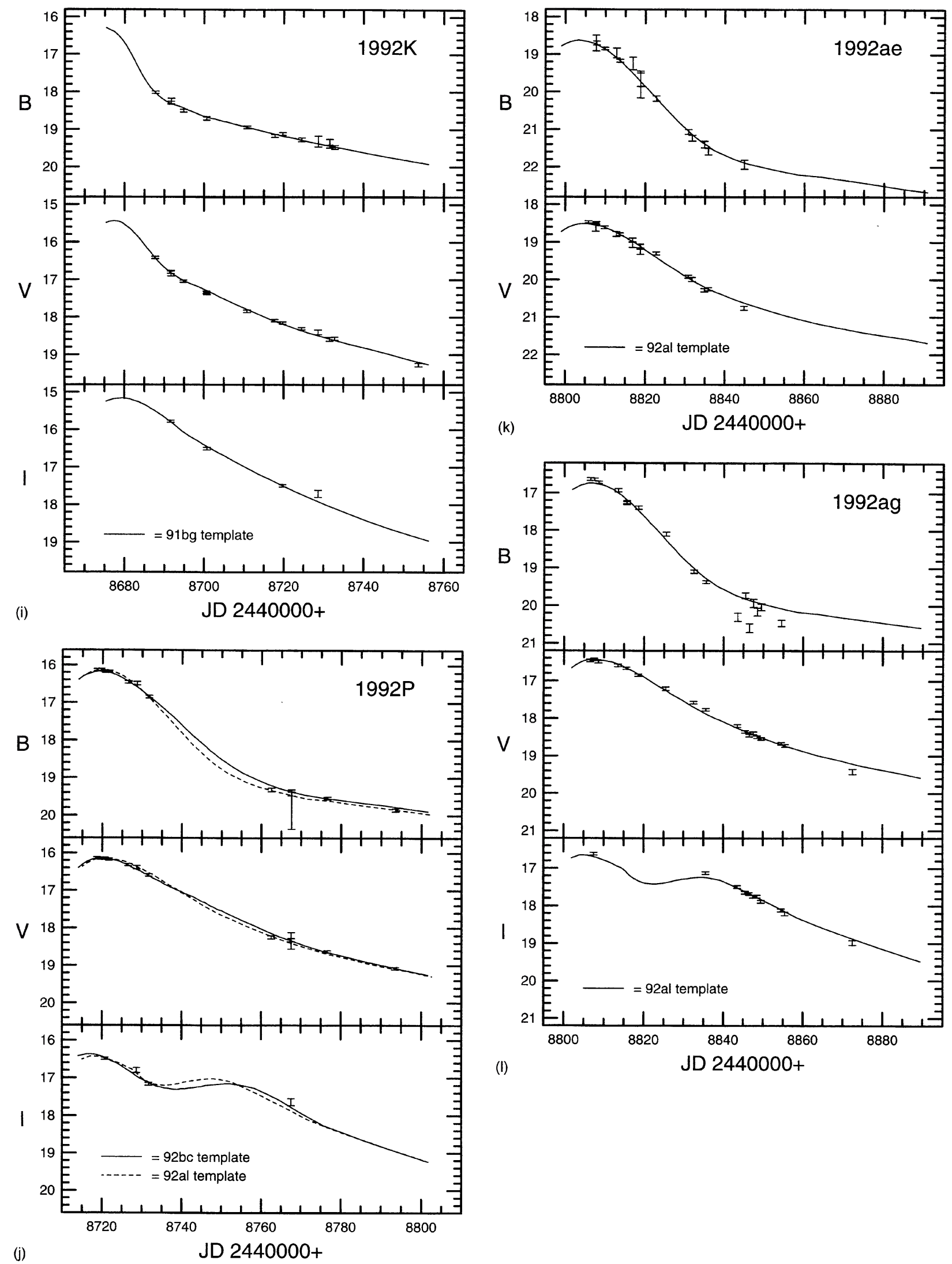

FIG. 3. (continued) 

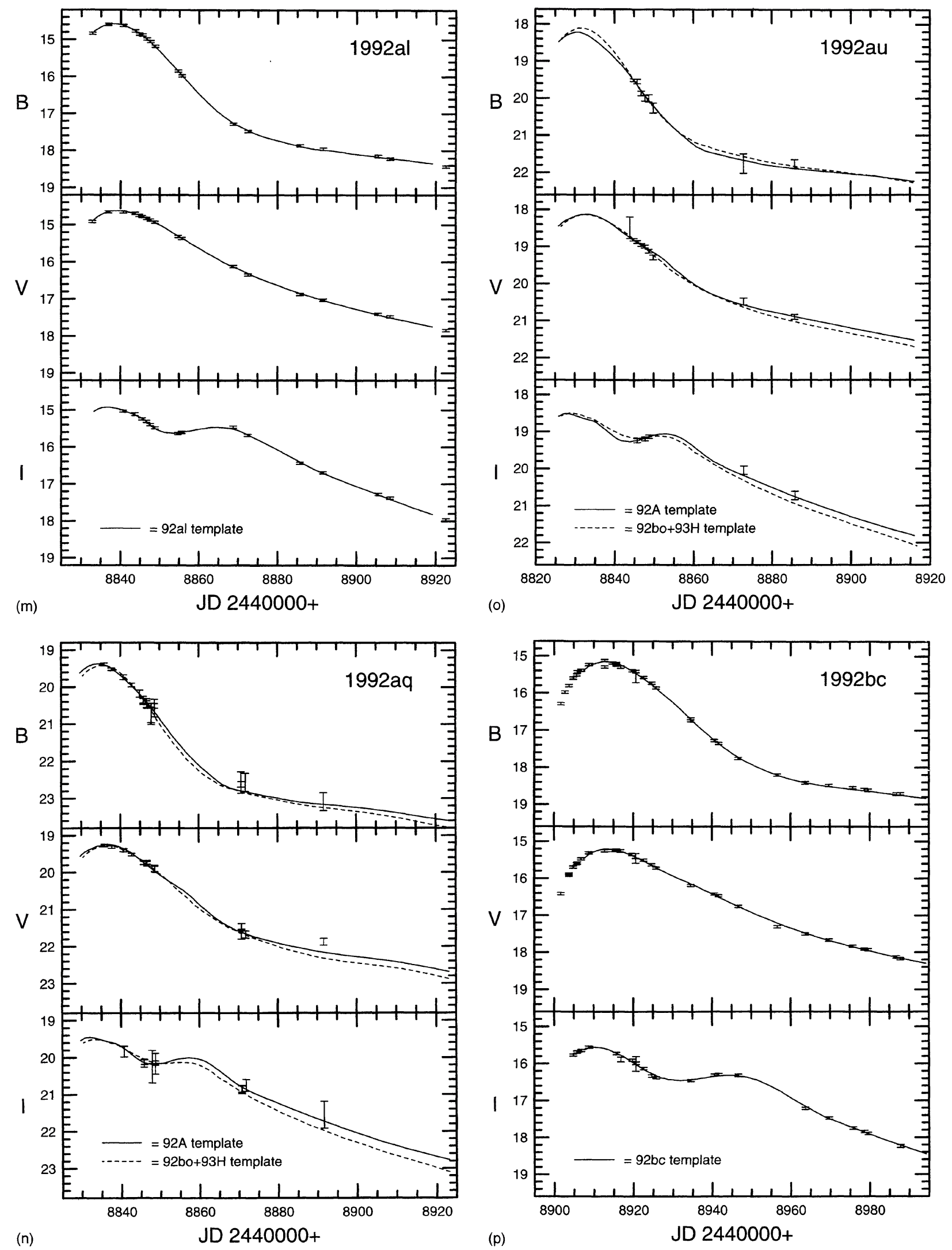

FIG. 3. (continued) 

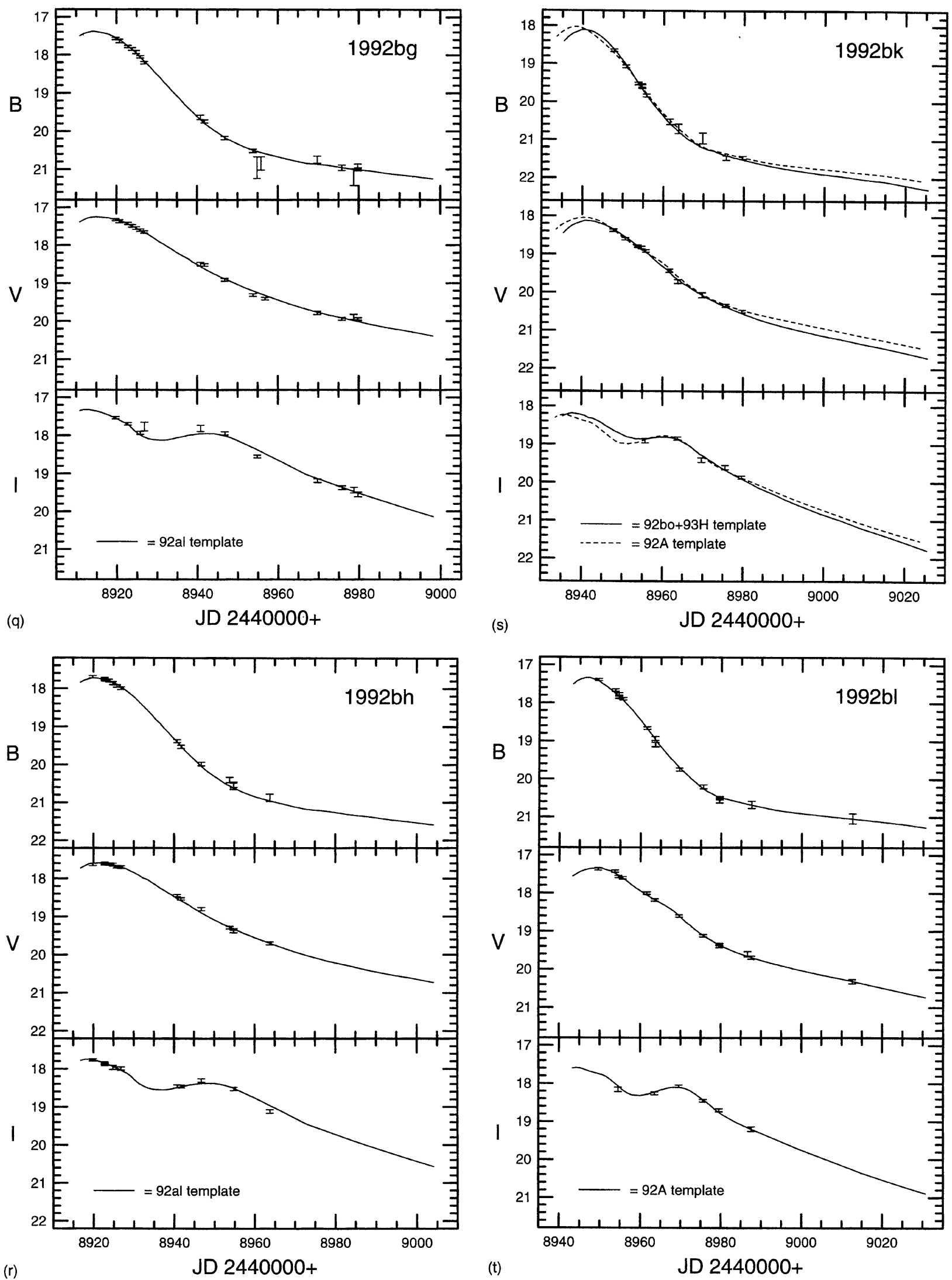

FIG. 3. (continued) 

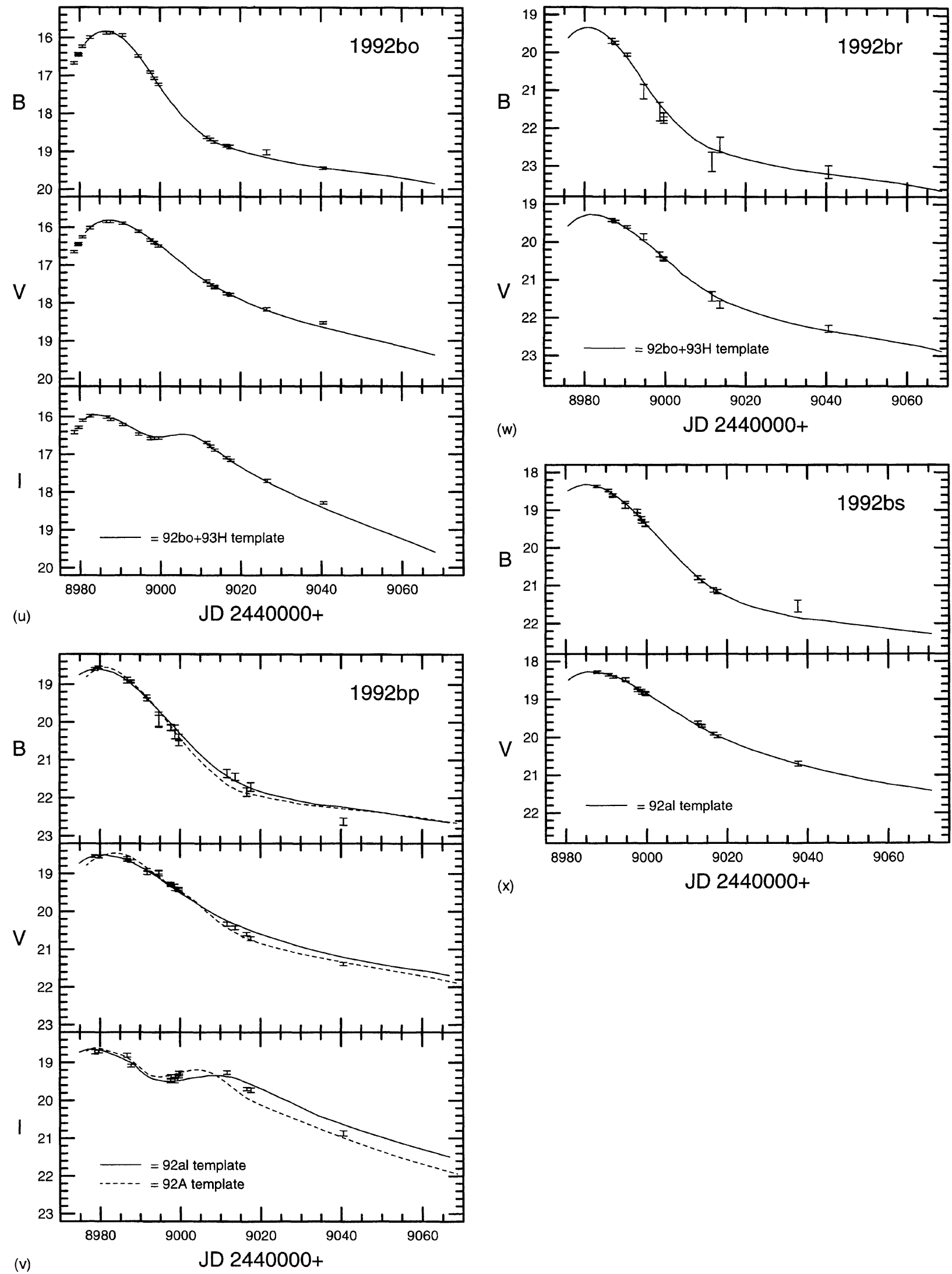

(x)

FIG. 3. (continued) 

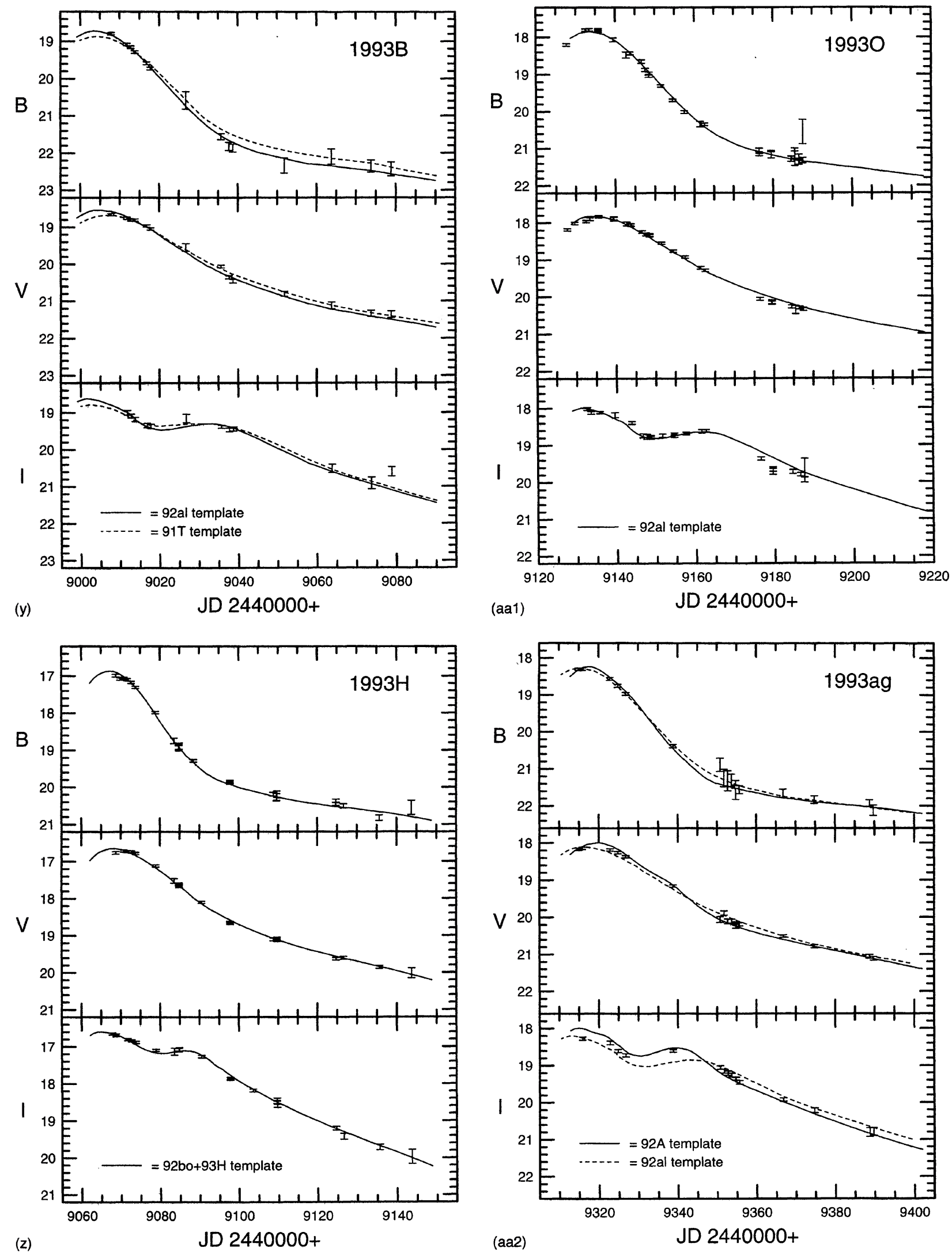

Fig. 3. (continued) 


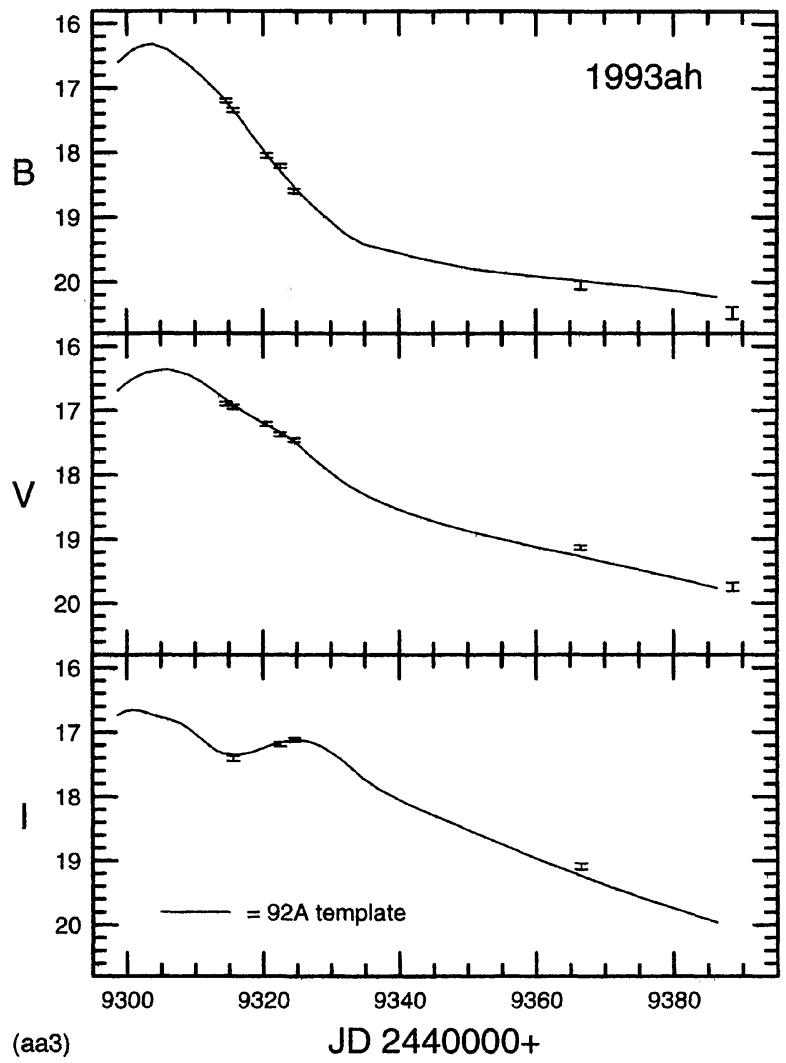

FIG. 3. (continued)

aging the results for the two templates. The corresponding errors were taken to be the greater of (a) half of the difference between the peak magnitude estimates of the two templates, (b) the $2 \sigma$ formal errors of the $\chi^{2}$ fits, or (c) $0.05^{m}$. When the difference in the reduced $\chi^{2}$ values was $>1.5$, the maximum-light magnitudes were taken from the single-bestfitting template, with the adopted error being the larger of the $2 \sigma$ formal error of the $\chi^{2}$ fit or $0.05^{m}$. Although these rules produced reasonable error estimates in most cases, we found that the errors derived for some $\mathrm{SNe}$ whose first light curve observations did not begin until $\sim 2$ weeks after maximum were unrealistically low. Hence, in all cases where template fits indicated that the first photometry was not obtained until $\geqslant 10$ days after $B$ maximum, we adopted the following error estimates: $0.2^{m}$ in $B, 0.15^{m}$ in $V$, and $0.15^{m}$ in $I$.

For each of the $29 \mathrm{SNe}$ in our sample, we also estimated the decline rate parameter $\Delta m_{15}(B)$ (Phillips 1993) which corresponds to the amount in magnitudes that the $B$ light curve decreases in brightness during the first 15 days after maximum. This parameter could be measured directly for the five best-observed SNe in the sample (1990af, 1992al, 1992bc, 1992bo, and 19930). For the remaining events, $\Delta m_{15}(B)$ was estimated by fitting a parabola to the reduced $\chi^{2}$ values yielded by the six template fits (see Paper IV for further details of this procedure). Note that when the smallest value of the reduced $\chi^{2}$ corresponded to either of the two extremes of the range of $\Delta m_{15}(B)$ represented by our tem-

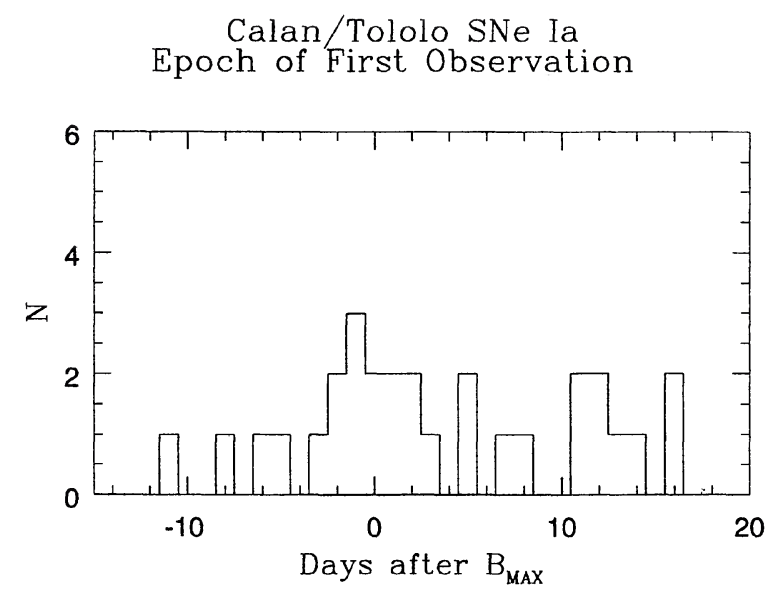

FIG. 4. Histogram showing the time with respect to $B_{M A X}$ of the first photometric observation in $B, V$, or $I$. Note that for nearly one third of the $\mathrm{SNe}$ the photometric monitoring started before or at maximum light.

plates (SN 1992bc with $\Delta m_{15}(B)=0.87$ and SN 1991bg with $\left.\Delta m_{15}(B)=1.93\right)$, we set the inferred value of $\Delta m_{15}(B)$ to the same value as the template rather than attempting to extrapolate a value.

Table 5 summarizes the resulting light curve parameters for all $29 \mathrm{SNe}$ Ia. Specifically, we give the epoch of $B$ maximum; the time with respect to $B_{M A X}$ of the first photometric observation in $B, V$, or $I$; the decline rate parameter $\Delta m_{15}(B)$; the apparent maximum-light magnitudes in $B, V$, and $I$; and the method employed to estimate the peak magnitudes where Data means that the values were measured directly from the photometry, Single Fit indicates that the best-fitting template was used, and Average signifies that the results from the two best template fits were averaged. In Fig. 4 we plot a histogram of the time with respect to $B_{M A X}$ of the first photometric observation in $B, V$, or $I$ for the 29 Calán/ Tololo SNe Ia.

In Table 6, we repeat the $\Delta m_{15}(B)$ values and give our final estimates of the peak magnitudes after correction for the extinction due to our own Galaxy (see Table 1) and the $K$ term. The uncertainties in the corrected magnitudes include errors in the observed magnitude (listed in Table 5), foreground reddening $\left(0.06^{m}\right.$ in $B, 0.045^{m}$ in $V$, and $0.03^{m}$ in $\left.I\right)$, as well as in the $K$ term (assumed to be $\pm 0.02^{m}$ ). We also list the "color" of the SN, $B_{M A X}-V_{M A X}$. (Note that, strictly speaking, this is not a color since $B_{M A X}$ and $V_{M A X}$ occur at slightly different times.) The uncertainties in the color were estimated in the following manner: (a) for the $11 \mathrm{SNe}$ for which the photometry started before maximum light we adopted an error of $0.03^{m}$ when the peak was very well observed (6 cases), or $0.05^{m}$ otherwise (5 cases), (b) when the coverage of the light curve started between days 1 and 10 (counted since $B_{M A X}$ ) the adopted error was the larger of half of the difference between the color estimates of the two templates or $0.05^{m}$; if the single-best fitting template technique was used we adopted an error of $0.05^{m}$, or (c) when the observations started after day 10 (counted since $B_{M A X}$ ) the adopted error was the larger of half of the difference between the color estimates of the two templates or $0.10^{m}$; if the 
TABLE 5. Light curve parameters.

\begin{tabular}{|c|c|c|c|c|c|c|c|}
\hline SN & $\begin{array}{c}T_{\max }(B) \\
2440000+\end{array}$ & 1st Obs. & $\Delta m_{15}(B)$ & $B_{\mathrm{MAX}}$ & $V_{\text {MAX }}$ & $I_{\mathrm{MAX}}$ & Method \\
\hline 19900 & $8076.96(43)$ & 0 & $0.96(10)$ & $16.59(08)$ & $16.51(06)$ & $16.80(08)$ & Average \\
\hline $1990 \mathrm{~T}$ & $8082.84(45)$ & +16 & $1.15(10)$ & $17.27(20)$ & $17.19(15)$ & $17.36(15)$ & Single Fit \\
\hline 1990Y & $8116.87(67)$ & +16 & $1.13(10)$ & $17.69(20)$ & $17.35(15)$ & $17.57(15)$ & Average \\
\hline 1990af & $8195.90(40)$ & -3 & $1.56(05)$ & $17.91(03)$ & $17.84(03)$ & $\cdots$ & Data \\
\hline $1991 S$ & $8348.04(118)$ & +13 & $1.04(10)$ & $17.78(20)$ & $17.75(15)$ & $17.99(15)$ & Average \\
\hline $1991 U$ & $8355.60(91)$ & +11 & $1.06(10)$ & $16.67(20)$ & $16.54(15)$ & $16.62(15)$ & Average \\
\hline 1991ag & $8413.17(216)$ & +7 & $0.87(10)$ & $14.67(13)$ & $14.58(14)$ & $14.83(19)$ & Average \\
\hline $1992 J$ & $8672.54(78)$ & +14 & $1.56(10)$ & $17.88(20)$ & $17.71(15)$ & $17.88(15)$ & Average \\
\hline $1992 \mathrm{~K}$ & $8675.41(51)$ & +12 & $1.93(10)$ & $16.30(20)$ & $15.44(15)$ & $15.16(15)$ & Single Fit \\
\hline $1992 \mathrm{P}$ & $8719.68(49)$ & -1 & $0.87(10)$ & $16.14(03)$ & $16.15(03)$ & $16.40(05)$ & Data $^{a}$ \\
\hline $1992 \mathrm{ae}$ & $8804.40(102)$ & +1 & $1.28(10)$ & $18.64(10)$ & $18.51(06)$ & $\ldots$ & Single Fit \\
\hline 1992ag & $8807.09(55)$ & -1 & $1.19(10)$ & $16.64(05)$ & $16.45(05)$ & $16.50(05)$ & Data $^{b}$ \\
\hline 1992al & $8838.36(40)$ & -5 & $1.11(05)$ & $14.59(03)$ & $14.64(03)$ & $14.93(05)$ & Data $^{c}$ \\
\hline $1992 \mathrm{aq}$ & $8835.58(46)$ & 0 & $1.46(10)$ & $19.39(07)$ & $19.26(05)$ & $19.48(08)$ & Average \\
\hline $1992 \mathrm{au}$ & $8831.54(84)$ & +12 & $1.49(10)$ & $18.17(20)$ & $18.13(15)$ & $18.51(15)$ & Average \\
\hline $1992 \mathrm{bc}$ & $8912.62(40)$ & -11 & $0.87(05)$ & $15.15(03)$ & $15.23(03)$ & $15.56(03)$ & Data \\
\hline 1992bg & $8915.00(68)$ & +5 & $1.15(10)$ & $17.39(05)$ & $17.25(05)$ & $17.32(05)$ & Single Fit \\
\hline $1992 \mathrm{bh}$ & $8920.92(46)$ & -1 & $1.05(10)$ & $17.68(05)$ & $17.60(03)$ & $17.75(05)$ & Data $^{c}$ \\
\hline $1992 \mathrm{bk}$ & $8939.80(90)$ & +8 & $1.57(10)$ & $18.07(08)$ & $18.08(05)$ & $18.21(05)$ & Average \\
\hline 1992bl & $8947.75(43)$ & +2 & $1.51(10)$ & $17.34(05)$ & $17.34(05)$ & $17.59(05)$ & Single Fit \\
\hline 1992bo & $8986.44(40)$ & -8 & $1.69(05)$ & $15.85(03)$ & $15.84(03)$ & $15.95(03)$ & Data \\
\hline $1992 b p$ & $8981.17(94)$ & -2 & $1.32(10)$ & $18.53(03)$ & $18.53(03)$ & $18.68(05)$ & Data $^{b}$ \\
\hline $1992 \mathrm{br}$ & $8981.32(120)$ & +5 & $1.69(10)$ & $19.34(16)$ & $19.27(09)$ & $\cdots$ & Single Fit \\
\hline $1992 \mathrm{bs}$ & $8985.87(75)$ & +2 & $1.13(10)$ & $18.33(06)$ & $18.27(05)$ & $\cdots$ & Single Fit \\
\hline 1993B & $9004.85(62)$ & +3 & $1.04(10)$ & $18.81(09)$ & $18.61(07)$ & $18.71(09)$ & Average \\
\hline $1993 \mathrm{H}$ & $9067.22(40)$ & +1 & $1.69(10)$ & $16.99(05)$ & $16.72(03)$ & $16.60(05)$ & Data $^{\mathrm{c}}$ \\
\hline 19930 & $9133.98(40)$ & -6 & $1.22(05)$ & $17.79(03)$ & $17.84(03)$ & $17.99(05)$ & Data $^{\mathrm{b}}$ \\
\hline 1993ag & $9316.91(116)$ & -2 & $1.32(10)$ & $18.30(05)$ & $18.12(03)$ & $18.22(05)$ & Data $^{\mathrm{b}}$ \\
\hline 1993ah & $9303.87(86)$ & +11 & $1.30(10)$ & $16.32(20)$ & $16.36(15)$ & $16.65(15)$ & Single Fit \\
\hline
\end{tabular}

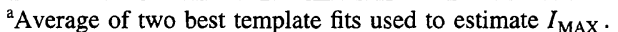

${ }^{b}$ Best-fitting template adjusted to first data point to estimate $I_{\mathrm{MAX}}$.

${ }^{\mathrm{c}}$ Best-fitting template used to estimate $I_{\mathrm{MAX}}$.

TABLE 6. Final corrected magnitudes and colors.

\begin{tabular}{|c|c|c|c|c|c|}
\hline $\mathrm{SN}$ & $\Delta m_{15}(B)$ & $B_{\mathrm{MAX}}$ & $V_{\text {MAX }}$ & $I_{\mathrm{MAX}}$ & $B_{\mathrm{MAX}}-V_{\mathrm{MAX}}$ \\
\hline 19900 & $0.96(10)$ & $16.32(10)$ & $16.31(08)$ & $16.70(09)$ & $0.01(05)$ \\
\hline $1990 \mathrm{~T}$ & $1.15(10)$ & $17.16(21)$ & $17.12(16)$ & $17.35(15)$ & $0.04(10)$ \\
\hline $1990 Y$ & $1.13(10)$ & $17.70(21)$ & $17.37(16)$ & $17.61(15)$ & $0.33(10)$ \\
\hline 1990af & $1.56(05)$ & $17.87(07)$ & $17.82(06)$ & $\cdots$ & $0.05(03)$ \\
\hline 1991S & $1.04(10)$ & $17.81(21)$ & $17.77(16)$ & $18.07(15)$ & $0.04(10)$ \\
\hline $1991 U$ & $1.06(10)$ & $16.40(21)$ & $16.34(16)$ & $16.52(15)$ & $0.06(10)$ \\
\hline 1991ag & $0.87(10)$ & $14.62(14)$ & $14.54(15)$ & $14.86(19)$ & $0.08(05)$ \\
\hline 1992J & $1.56(10)$ & $17.70(21)$ & $17.58(16)$ & $17.84(15)$ & $0.12(10)$ \\
\hline $1992 \mathrm{~K}$ & $1.93(10)$ & $15.83(21)$ & $15.09(16)$ & $14.94(15)$ & $0.74(10)$ \\
\hline $1992 \mathrm{P}$ & $0.87(10)$ & $16.08(07)$ & $16.11(06)$ & $16.39(06)$ & $-0.03(03)$ \\
\hline 1992ae & $1.28(10)$ & $18.62(12)$ & $18.51(08)$ & $\cdots$ & $0.11(05)$ \\
\hline 1992ag & $1.19(10)$ & $16.41(08)$ & $16.28(07)$ & $16.41(06)$ & $0.13(05)$ \\
\hline 1992al & $1.11(05)$ & $14.60(07)$ & $14.65(06)$ & $14.94(06)$ & $-0.05(03)$ \\
\hline 1992aq & $1.46(10)$ & $19.45(09)$ & $19.35(07)$ & $19.77(09)$ & $0.10(05)$ \\
\hline 1992au & $1.49(10)$ & $18.21(21)$ & $18.16(16)$ & $18.41(15)$ & $0.05(10)$ \\
\hline $1992 b c$ & $0.87(05)$ & $15.16(07)$ & $15.24(06)$ & $15.58(05)$ & $-0.08(03)$ \\
\hline $1992 b g$ & $1.15(10)$ & $16.72(08)$ & $16.76(07)$ & $17.04(06)$ & $-0.04(05)$ \\
\hline $1992 \mathrm{bh}$ & $1.05(10)$ & $17.70(08)$ & $17.62(06)$ & $17.80(06)$ & $0.08(05)$ \\
\hline 1992bk & $1.57(10)$ & $18.11(10)$ & $18.11(07)$ & $18.31(06)$ & $0.00(05)$ \\
\hline 1992bl & $1.51(10)$ & $17.36(08)$ & $17.36(07)$ & $17.64(06)$ & $0.00(05)$ \\
\hline 1992bo & $1.69(05)$ & $15.86(07)$ & $15.85(06)$ & $15.97(05)$ & $0.01(03)$ \\
\hline $1992 b p$ & $1.32(10)$ & $18.41(07)$ & $18.46(06)$ & $18.78(06)$ & $-0.05(05)$ \\
\hline $1992 \mathrm{br}$ & $1.69(10)$ & $19.38(17)$ & $19.34(10)$ & $\cdots$ & $0.04(05)$ \\
\hline $1992 \mathrm{bs}$ & $1.13(10)$ & $18.37(09)$ & $18.30(07)$ & $\cdots$ & $0.07(05)$ \\
\hline 1993B & $1.04(10)$ & $18.53(11)$ & $18.41(09)$ & $18.70(10)$ & $0.12(05)$ \\
\hline $1993 \mathrm{H}$ & $1.69(10)$ & $16.84(08)$ & $16.61(06)$ & $16.55(06)$ & $0.23(05)$ \\
\hline 19930 & $1.22(05)$ & $17.67(07)$ & $17.76(06)$ & $17.99(06)$ & $-0.09(03)$ \\
\hline 1993ag & $1.32(10)$ & $17.72(08)$ & $17.69(06)$ & $18.01(06)$ & $0.03(05)$ \\
\hline 1993ah & $1.30(10)$ & $16.33(21)$ & $16.37(16)$ & $16.68(15)$ & $-0.04(10)$ \\
\hline
\end{tabular}


single-best fitting template technique was used we adopted an error of $0.10^{m}$.

This paper was possible thanks to grant 92/0312 from Fondo Nacional de Ciencias y Tecnología (FONDECYT-Chile). M.H. acknowledges support provided for this work by the National Science Foundation through grant number GF-1002-96 from the Association of Universities for Research in Astronomy, Inc., under NSF Cooperative Agree ment No. AST-8947990 and from Fundación Andes under project C-12984. J.M. and M.H. acknowledge support by Cátedra Presidencial de Ciencias 1996-1997.

\section{REFERENCES}

Burstein, D., \& Heiles, C. 1982, AJ, 87, 1165

Hamuy, M., et al. 1993a, AJ, 106, 2392 (Paper I)

Hamuy, M., Phillips, M. M., Wells, L. A., \& Maza, J. 1993b, PASP, 105, 787

Hamuy, M., et al. 1994, AJ, 108, 2226 (Paper III)

Hamuy, M., Phillips, M. M., Maza, J., Suntzeff, N. B., Schommer, R. A., \& Avilés, R. 1995, AJ, 109, 1 (Paper IV)

Hamuy, M., Phillips, M. M., Schommer, R. A., Suntzeff, N. B., Maza,
J., \& Avilés, R. 1996a, AJ, 112, 2391 (Paper V)

Hamuy, M., Phillips, M. M., Suntzeff, N. B., Schommer, R. A., Maza, J., \& Avilés, R. 1996b, AJ, 112, 2398 (Paper VI)

Hamuy, M., Phillips, M. M., Suntzeff, N. B., Schommer, R. A., Maza, J., Smith, R. C., Lira, P., \& Avilés, R. 1996c, 112, 2438 (Paper VIII)

Maza, J., Hamuy, M., Phillips, M. M., Suntzeff, N. B., \& Avilés, R. 1994, ApJ, 424, L107 (Paper II)

Phillips, M. M. 1993, ApJ, 413, L105 